Pacific

Journal of

Mathematics

AN INVERSE PROBLEM FOR THE TRANSPORT EQUATION IN THE PRESENCE OF A RIEMANNIAN METRIC

Stephen R. McDowall 


\title{
AN INVERSE PROBLEM FOR THE TRANSPORT EQUATION IN THE PRESENCE OF A RIEMANNIAN METRIC
}

\author{
Stephen R. MCDowall
}

\begin{abstract}
The stationary linear transport equation models the scattering and absorption of a low-density beam of neutrons as it passes through a body. In Euclidean space, to a first approximation, particles travel in straight lines. Here we study the analogous transport equation for particles in an ambient field described by a Riemannian metric where, again to first approximation, particles follow geodesics of the metric. We consider the problem of determining the scattering and absorption coefficients from knowledge of the albedo operator on the boundary of the domain. Under certain restrictions, the albedo operator is shown to determine the geodesic ray transform of the absorption coefficient; for "simple" manifolds this transform is invertible and so the coefficient itself is determined. In dimensions 3 or greater, we show that one may then obtain the collision (or scattering) kernel.
\end{abstract}

\section{Introduction}

The stationary linear transport equation models the time-independent scattering of a low-density beam of particles off a higher-density material. The term "linear" refers to the fact that the equation models only scattering of particles from the material and assumes that the density of particles is low enough that particle-to-particle interaction may be neglected. If $(x, v)$ is a point in phase space we denote by $f(x, v)$ the density of particles at position $x$ with velocity $v$. While $f$ is strictly speaking a density, for large numbers of particles it is reasonable to represent $f$ as an $L^{1}$ function. In free-space, $f$ satisfies the transport equation

$$
-v \cdot \nabla_{x} f(x, v)-\sigma_{a}(x, v) f(x, v)+\int_{V} k\left(x, v^{\prime}, v\right) f\left(x, v^{\prime}\right) d v^{\prime}=0 .
$$

The first term describes the straight-line motion of a particle that does not interact with the material. The second term represents the loss of a particle at $(x, v)$ due to scattering to another velocity or due to absorption, quantified by the function $\sigma_{a}(x, v)$. The final term accounts for the production of a particle at $(x, v)$ due to scattering from other directions; the kernel $k\left(x, v^{\prime}, v\right)$ 
represents the probability of a particle at $\left(x, v^{\prime}\right)$ scattering to $(x, v)$. The reader is referred to $[\mathbf{R S}]$ for a detailed explanation.

We are concerned here with the situation of particles moving in an ambient field represented by a Riemannian metric. The principal departure from (1) is that in the absence of interaction, a particle will follow the geodesics of the metric. We shall study the problem of determining the absorption coefficient $\sigma_{a}(x, v)$ and the collision kernel $k\left(x, v^{\prime}, v\right)$ from knowledge of the positions and velocities of particles entering and leaving a bounded body.

Let $M \subset \mathbb{R}^{n}, n \geq 2$, be a bounded domain with $C^{\infty}$ boundary; let $g$ be a Riemannian metric on $M$. We shall put restrictions on the metric $g$ in due course. Define the incoming and outgoing bundles on $\partial M$ as

$$
\Gamma_{ \pm}=\left\{(x, v) \in T M \mid x \in \partial M, \pm\langle v, \nu\rangle_{g_{x}}>0\right\},
$$

where $\nu$ is the outward unit normal vector to $\partial M$ and $\langle\cdot, \cdot\rangle_{g_{x}}$ is the inner product with respect to the metric $g$. We shall also use $\|\cdot\|_{g_{x}}$ to denote the norm with respect to $g$. If $(x, v) \in T M$ we shall denote by $\gamma_{(x, v)}(t)$ the geodesic satisfying $\gamma_{(x, v)}(0)=x$ and $\dot{\gamma}_{(x, v)}(0)=v$; we introduce the compressed notation

$$
\vec{\gamma}_{(x, v)}(t)=\left(\gamma_{(x, v)}(t), \dot{\gamma}_{(x, v)}(t)\right)
$$

For $v \neq 0$, define the time-to-boundary functions $\tau_{ \pm}: T M \rightarrow \mathbb{R}^{+}$by

$$
\tau_{ \pm}(x, v)=\min \left\{t>0 \mid \gamma_{(x, v)}( \pm t) \in \partial M\right\}
$$

and set $\tau(x, v)=\tau_{-}(x, v)+\tau_{+}(x, v)$. While in general $\tau_{ \pm}$might be infinite, we will place restrictions on the metric that ensure that $\tau_{ \pm}$are well-defined and finite.

With these preparations we are able to generalize Equation (1) and state the results of the paper. Denote by $\mathcal{D}$ the derivative along the geodesic flow,

$$
\mathcal{D} f(x, v)=\left.\frac{\partial}{\partial t}\right|_{t=0} f\left(\gamma_{(x, v)}(t), \dot{\gamma}_{(x, v)}(t)\right) .
$$

If $\left(x^{i}, y^{i}\right)_{i=1}^{n}$ are local coordinates for $T M$ with the $\left(y^{i}\right)$ with respect to the natural basis $\left(\frac{\partial}{\partial x^{i}}\right)$, we have in these coordinates

$$
\mathcal{D} f=\frac{\partial f}{\partial x^{i}} y^{i}+\frac{\partial f}{\partial y^{i}}\left(-y^{j} y^{k} \Gamma_{j k}^{i}\right),
$$

where $\Gamma_{j k}^{i}$ are the Christoffel symbols of the Levi-Civita connection of $g$. The transport equation (1) is replaced in our setting by

$$
-\mathcal{D} f(x, v)-\sigma_{a}(x, v) f(x, v)+\int_{T_{x} M} k\left(x, v^{\prime}, v\right) f\left(x, v^{\prime}\right) d v_{x}^{\prime}=0 .
$$

The measure $d v_{x}$ is the Euclidean volume form on $T_{x} M$ determined by the metric $g_{x}$ at $x$ (see Definition 2.1). Given a function $f_{-}(x, v)$ on $\Gamma_{-}$let $f$ be 
the solution to (2) with boundary condition $\left.f\right|_{\Gamma_{-}}=f_{-}$, assuming it exists. One may then define the albedo operator

$$
\mathcal{A}:\left.f_{-} \mapsto f\right|_{\Gamma_{+}} .
$$

The inverse problem addressed here is the unique determination of $\sigma_{a}(x, v)$ and $k\left(x, v^{\prime}, v\right)$ for all $x \in M$ and $v, v^{\prime} \in T_{x} M$, from the knowledge of $\mathcal{A}$. The main results are the content of Theorems 4.2 and 4.4, which state that under the assumption of simplicity of the metric (see below) and a priori assumptions $(3),(4)$ on the coefficients themselves, we can determine $\sigma_{a}$ in dimensions $n \geq 2$ and $k$ in dimensions $n \geq 3$.

We remark that if we assume that $k\left(x, v^{\prime}, v\right)=0$ for all $\left\|v^{\prime}\right\|_{g_{x}} \neq\|v\|_{g_{x}}$ that is, that all scattering occurrences preserve speed - then we may consider the problem on the unit sphere bundle $\Omega M$ of $M$. We replace $\Gamma_{ \pm}$ by their equivalents restricted to unit tangent vectors, and the integration in (2) is taken over $\Omega_{x} M$, the unit sphere in $T_{x} M$. The analysis and results of this paper remain valid in this setting. Indeed, under this assumption on $k$ we may consider $\Gamma_{ \pm}$defined to include tangent vectors of lengths $0<a \leq\|v\| \leq b<\infty$ and in (2) integrate over the corresponding annular region in $T_{x} M$. Once again the results remain valid in this setting.

When the ambient metric is Euclidean, the inverse problem for (1) was considered in [CS2] and we shall approach the problem here in the same manner. In [CS2] it is shown that the most singular part of the distributional kernel of the albedo operator determines the x-ray transform of the absorption coefficient. The next most singular part determines the collision kernel $k$ in dimensions 3 and greater. Here we shall show that the first term determines the geodesic ray transform of $\sigma_{a}$, namely the integrals of $\sigma_{a}$ along geodesics of $g$. In order to recover $\sigma_{a}$ we make the restrictive assumption that the metric $g$ be "simple." This means that $M$ is strictly convex with respect to $g$ and that for any $x \in \bar{M}$ the exponential map $\operatorname{Exp}_{x}: \operatorname{Exp}_{x}^{-1}(\bar{M}) \rightarrow \bar{M}$ is a diffeomorphism. This assumption, together with the full set of geodesics joining boundary points, ensures that the ray transform is invertible (see $[\mathbf{B G}]$ and $[\mathbf{M}]$, and $[\mathbf{S h} \mathbf{1}]$ for an extensive treatment of the ray transform).

In the Riemannian setting, an inverse source problem for the stationary transport equation is addressed in [Sh2] (see also [Sh1]), where $k\left(x, v, v^{\prime}\right)=$ $k\left(x,\left\langle v, v^{\prime}\right\rangle\right)$ is assumed to depend on the angle between $v$ and $v^{\prime}$ and the object of interest is the reconstruction of an isotropic source term. In $[\mathbf{F}]$ the (time-dependent) radiative transfer equation is derived for a medium with spatially varying refractive index and with scattering kernel $k$ independent of position. Such a refractive index is represented by a Riemannian metric.

More is known when the metric is assumed to be Euclidean. The timedependent inverse problem was treated in $[\mathbf{C S 1}]$ and the stationary case in [CS2]. Stability estimates based on this work were obtained under certain restrictions in $[\mathbf{W}]$. 
The stationary transport equation is used to model the absorption and scattering of near-infrared light; the determination of the absorption and scattering properties of a medium from the measurement of the response to such transmitted light is known as optical tomography and has been applied to the problems of medical imaging $([\mathbf{A}])$. For two-dimensional domains, recent works include [T2], where a homogeneous collision kernel $k$ that is a function of two independent directions was shown to be uniquely determined; [SU], in which the assumption on homogeneity is dropped and $k$ is assumed to be small relative to $\sigma_{a}$, with an explicit constant given; and [T1], where the smallness is removed in the case of weakly anisotropic scattering. In [SU] the authors prove also a stability estimate; further stability results may be found in $[\mathbf{R}]$. Note that some kind of smallness does need to be assumed on $k$, to ensure that the production rate is in some sense less than the absorption rate, thus keeping the energy of the system bounded.

This paper is organized as follows: in Section 2 we state our assumptions precisely, prove solvability of the forward problem given these assumptions, and demonstrate well-definedness of the boundary albedo operator. In Section 3 we construct distribution solutions to (2) with delta-type boundary conditions. This facilitates determination of the distribution kernel of the albedo operator as the sum of three terms of differing singularity strengths. In Section 4 we prove that it is possible to extract from the kernel each of the singular terms and that these determine the absorption coefficient (in all dimensions) and the collision kernel (in dimensions 3 and greater).

\section{The forward problem and the albedo operator}

We begin by defining the volume form on $T M$ :

Definition 2.1. The Liouville volume form is the canonical $2 n$-form defined on $T M$ that is preserved under the geodesic flow of $g$. It is the product of the Riemannian volume form $d \omega(x)$ on the manifold $M$ and the Euclidean volume form $d v_{x}$ defined in the tangent space $T_{x} M$ by the metric $g_{x}$ at $x \in M$ (see $[\mathbf{K H}]$, for example).

The sense in which the definition above holds is the following: given an orthonormal basis $Y_{1}, \ldots, Y_{n}$ for $T_{x} M$, extend it to an adapted basis of vector fields in a neighborhood of $x$ by parallel transport with respect to the LeviCivita connection of $g$. If $d y^{j}$ are the forms dual to $Y_{j}$, the Liouville form is given by $\sqrt{\operatorname{det} g} d x^{1} \wedge \cdots \wedge d x^{n} \wedge d y^{1} \wedge \cdots \wedge d y^{n}$. For a fixed chart one can in principle express this form in terms of the natural basis $\frac{\partial}{\partial x^{1}}, \cdots \frac{\partial}{\partial x^{n}}$, and to do so one must solve the system of differential equations describing the evolution of the $Y_{j}$ in terms of the $\frac{\partial}{\partial x^{j}}$ as the basepoint $x$ for $T_{x} M$ varies. The interested reader can consult Equations (6) and (7) on page 47 of $[\mathbf{H}]$. It is not possible to express the Liouville form at an arbitrary point in a chart 
without reference to a basepoint. We set $L^{1}(T M)=L^{1}\left(T M, d v_{x} d \omega(x)\right)$ and $L^{1}\left(T_{x} M\right)=L^{1}\left(T_{x} M, d v_{x}\right)$.

We assume that the pair $\left(\sigma_{a}, k\right)$ is admissible (see $[\mathbf{R S}]$ ), namely that

$$
\left\{\begin{array}{l}
0 \leq \sigma_{a} \in L^{\infty}(T M), \\
0 \leq k\left(x, v^{\prime}, \cdot\right) \in L^{1}\left(T_{x} M\right) \text { for a.e. }\left(x, v^{\prime}\right) \in T M, \\
\sigma_{p}\left(x, v^{\prime}\right)=\int_{T_{x} M} k\left(x, v^{\prime}, v\right) d v_{x} \in L^{\infty}(T M) .
\end{array}\right.
$$

Notice that the operators $f \mapsto \sigma_{a} f$ and $f \mapsto \int_{T_{x} M} k\left(x, v^{\prime}, v\right) f\left(x, v^{\prime}\right) d v_{x}^{\prime}$ are bounded on $L^{1}(T M)$.

Equation (2) may not be uniquely solvable, so we shall make the following subcriticality assumptions (see [RS]), which ensure that the problem is wellposed:

$$
\left\|\tau \sigma_{a}\right\|_{L^{\infty}(T M)}<\infty \quad \text { and } \quad\left\|\tau \sigma_{p}\right\|_{L^{\infty}(T M)}<1 .
$$

Furthermore, even in the Euclidean setting, without further restriction on $\sigma_{a}$ the albedo operator does not uniquely determine $\sigma_{a}$ (see [CS2]). To remove this lack of uniqueness, we assume that $\sigma_{a}$ depends only on the speed $\|v\|_{g_{x}}$, not on the direction:

$$
\sigma_{a}(x, v)=\sigma_{a}\left(x,\|v\|_{g_{x}}\right) .
$$

In order to pose the boundary value problem we must specify the volume form on $\left\{(x, v) \mid x \in \partial M, v \in T_{x} M\right\}$, in particular on $\Gamma_{ \pm}$. If $(x, v) \in T M$, define $\left(x^{\prime}, v^{\prime}\right) \in \Gamma_{-}$and $t \geq 0$ by

$$
x^{\prime}=\gamma_{(x, v)}\left(-\tau_{-}(x, v)\right), \quad v^{\prime}=\dot{\gamma}_{(x, v)}\left(-\tau_{-}(x, v)\right), \quad t=\tau_{-}(x, v),
$$

and define $F: \Gamma_{-} \times \mathbb{R} \rightarrow T M$ by

$$
F\left(x^{\prime}, v^{\prime}, t\right)=\left(\gamma_{\left(x^{\prime}, v^{\prime}\right)}(t), \dot{\gamma}_{\left(x^{\prime}, v^{\prime}\right)}(t)\right) .
$$

Define the product measure $d \mu\left(x^{\prime}, v^{\prime}\right) d t$ on $\Gamma_{-} \times \mathbb{R}$ by

$$
d \mu\left(x^{\prime}, v^{\prime}\right) d t=F^{*}\left(d v_{x} d \omega(x)\right),
$$

where $F^{*}$ is the pullback map defined by $F$. This measure is similarly defined on $\Gamma_{+}$. We denote $L^{1}\left(\Gamma_{ \pm}\right)=L^{1}\left(\Gamma_{ \pm}, d \mu\left(x^{\prime}, v^{\prime}\right)\right)$. The following lemma is immediate.

Lemma 2.2. If $f \in L^{1}(T M)$ then

$$
\int_{M} \int_{T_{x} M} f(x, v) d v_{x} d \omega=\int_{\Gamma_{ \pm}} \int_{0}^{\tau_{\mp}\left(x^{\prime}, v^{\prime}\right)} f\left(\gamma_{\left(x^{\prime}, v^{\prime}\right)}(t), \dot{\gamma}_{\left(x^{\prime}, v^{\prime}\right)}(t)\right) d t d \mu\left(x^{\prime}, v^{\prime}\right) .
$$

We define the function space $\mathcal{W}$ in which we shall prove solvability of (2) for boundary functions $f_{-} \in L^{1}\left(\Gamma_{-}\right)$. We shall see below that functions $f$ in $\mathcal{W}$ have a well-defined trace in $L^{1}\left(\Gamma_{+}\right)$. Let

$$
\mathcal{W}=\left\{f \mid \mathcal{D} f \in L^{1}(T M), \tau^{-1} f \in L^{1}(T M)\right\},
$$


with norm

$$
\|f\|_{\mathcal{W}}=\|\mathcal{D} f\|_{L^{1}(T M)}+\left\|\tau^{-1} f\right\|_{L^{1}(T M)} .
$$

The following trace theorem is proven in [CS2] for the Euclidean metric and we repeat a sketch of the proof in our setting.

Theorem 2.3. If $f(x, v) \in \mathcal{W}$ then

$$
\left\|\left.f\right|_{\Gamma_{ \pm}}\right\|_{L^{1}\left(\Gamma_{ \pm}, d \mu\right)} \leq\|\mathcal{D} f\|_{L^{1}(T M)}+\left\|\tau^{-1} f\right\|_{L^{1}(T M)} .
$$

Proof. Observe that if $h, h^{\prime} \in L^{1}([0, a])$, for some $a>0$, then

$$
|h(0)| \leq\left\|h^{\prime}\right\|_{L^{1}([0, a])}+\frac{1}{a}\|h\|_{L^{1}([0, a])} .
$$

Let $f$ be as in the hypothesis of the theorem and define

$$
h\left(t, x^{\prime}, v^{\prime}\right)=f\left(\vec{\gamma}_{\left(x^{\prime}, v^{\prime}\right)}(t)\right), \quad\left(x^{\prime}, v^{\prime}\right) \in \Gamma_{-}, \quad 0 \leq t \leq \tau_{+}\left(x^{\prime}, v^{\prime}\right) .
$$

Then

$$
\begin{aligned}
\mathcal{D} f\left(\vec{\gamma}_{\left(x^{\prime}, v^{\prime}\right)}(t)\right) & =\left.\frac{\partial}{\partial s}\right|_{s=0} f\left(\vec{\gamma}_{\left(\vec{\gamma}_{\left(x^{\prime}, v^{\prime}\right)}(t)\right)}(s)\right) \\
& =\left.\frac{\partial}{\partial s}\right|_{s=0} f\left(\vec{\gamma}_{\left(x^{\prime}, v^{\prime}\right)}(t+s)\right) \\
& =\partial_{t} h\left(t, x^{\prime}, v^{\prime}\right),
\end{aligned}
$$

so $\partial_{t} h \in L^{1}(T M)$; therefore, by Fubini's theorem, $\partial_{t} h \in L^{1}\left(\left[0, \tau_{+}\left(x^{\prime}, v^{\prime}\right)\right]\right)$ for a.e. $\left(x^{\prime}, v^{\prime}\right)$. Next,

$$
\begin{aligned}
\int_{0}^{\tau_{+}\left(x^{\prime}, v^{\prime}\right)}\left|h\left(s, x^{\prime}, v^{\prime}\right)\right| d s & \leq \int_{0}^{\tau_{+}\left(x^{\prime}, v^{\prime}\right)} \int_{0}^{s}\left|\partial_{t} h\left(t, x^{\prime}, v^{\prime}\right)\right| d t d s \\
& \leq \tau_{+}\left(x^{\prime}, v^{\prime}\right)\left\|\partial_{t} h\right\|_{L^{1}\left(\left[0, \tau_{+}\left(x^{\prime}, v^{\prime}\right)\right]\right)}
\end{aligned}
$$

so that $h\left(t, x^{\prime}, v^{\prime}\right)$ is also in $L^{1}\left(\left[0, \tau_{+}\left(x^{\prime}, v^{\prime}\right)\right]\right)$ for a.e. $\left(x^{\prime}, v^{\prime}\right)$. Thus

$$
\begin{aligned}
\left|f\left(x^{\prime}, v^{\prime}\right)\right| & =\mid f\left(\vec{\gamma}_{\left(x^{\prime}, v^{\prime}\right)}(0) \mid\right. \\
& \leq \int_{0}^{\tau_{+}\left(x^{\prime}, v^{\prime}\right)}\left|\mathcal{D} f\left(\vec{\gamma}_{\left(x^{\prime}, v^{\prime}\right)}(t)\right)\right| d t \\
& +\frac{1}{\tau_{+}\left(x^{\prime}, v^{\prime}\right)} \int_{0}^{\tau_{+}\left(x^{\prime}, v^{\prime}\right)}\left|f\left(\vec{\gamma}_{\left(x^{\prime}, v^{\prime}\right)}(t)\right)\right| d t .
\end{aligned}
$$

Integrating this inequality over $\Gamma_{-}$gives the result. The proof for $\Gamma_{+}$is similar.

Theorem 2.3 gives that the trace operator, $\left.f \mapsto f\right|_{\Gamma_{ \pm}}$, is continuous from $\mathcal{W}$ into $L^{1}\left(\Gamma_{ \pm}, d \mu\right)$. 
We proceed now to solve the boundary value problem (2) with $\left.f\right|_{\Gamma_{-}}=$ $f_{-} \in L^{1}\left(\Gamma_{-}, d \mu\right)$ and shall do so by reformulating the problem as an integral equation. First,

$$
\begin{aligned}
\mathcal{D} f(x, v) & =g(x, v) \\
\left.f\right|_{\Gamma_{-}} & =0
\end{aligned}
$$

has solution

$$
f(x, v)=\int_{0}^{\tau_{-}(x, v)} g\left(\vec{\gamma}_{(x, v)}\left(s-\tau_{-}(x, v)\right)\right) d s .
$$

To see this, we must calculate the seemingly intractable

$$
\mathcal{D} f(x, v)=\left.\frac{\partial}{\partial t}\right|_{t=0} \int_{0}^{\tau_{-}\left(\vec{\gamma}_{(x, v)}(t)\right)} g\left(\vec{\gamma}_{\left(\vec{\gamma}_{(x, v)}(t)\right)}\left(s-\tau_{-}\left(\vec{\gamma}_{(x, v)}(t)\right)\right)\right) d s .
$$

This simplifies considerably when one observes that

$$
\vec{\gamma}_{\left(\vec{\gamma}_{(x, v)}(t)\right)}(s)=\vec{\gamma}_{(x, v)}(s+t)
$$

and

$$
\tau_{-}\left(\vec{\gamma}_{(x, v)}(t)\right)=t+\tau_{-}(x, v)
$$

Thus

$$
\begin{aligned}
\mathcal{D} f & =\left.\frac{\partial}{\partial t}\right|_{t=0} \int_{0}^{t+\tau_{-}(x, v)} g\left(\vec{\gamma}_{(x, v)}\left(s-\tau_{-}(x, v)\right)\right) d s \\
& =g\left(\vec{\gamma}_{(x, v)}(0)\right)=g(x, v) .
\end{aligned}
$$

Lemma 2.4. Let $f_{-}$be a function on $\Gamma_{-}$. Then

$$
\begin{aligned}
\mathcal{D} f(x, v)+\sigma_{a}(x, v) f(x, v) & =0 \text { in } T M \\
\left.f\right|_{\Gamma_{-}} & =f_{-}
\end{aligned}
$$

has solution $J f_{-}$, where

$$
J f_{-}(x, v)=E\left(x, v, 0,-\tau_{-}(x, v)\right) f_{-}\left(\vec{\gamma}_{(x, v)}\left(-\tau_{-}(x, v)\right)\right),
$$

and where

$$
E(x, v, s, t)=\exp \left(\int_{s}^{t} \sigma_{a}\left(\vec{\gamma}_{(x, v)}(p)\right) d p\right) .
$$

Proof. We compute

$$
\begin{aligned}
\mathcal{D} E\left(x, v, 0,-\tau_{-}(x, v)\right) & =\left.\frac{\partial}{\partial t}\right|_{t=0} E\left(\vec{\gamma}_{(x, v)}(t), 0,-\tau_{-}\left(\vec{\gamma}_{(x, v)}(t)\right)\right) \\
& =\left.\frac{\partial}{\partial t}\right|_{t=0} \exp \left(\int_{0}^{-t-\tau_{-}(x, v)} \sigma_{a}\left(\vec{\gamma}_{(x, v)}(p+t) d p\right)\right. \\
& =-E\left(x, v, 0,-\tau_{-}(x, v)\right) \sigma_{a}(x, v)
\end{aligned}
$$


and

$$
\begin{aligned}
\mathcal{D} f_{-}\left(\vec{\gamma}_{(x, v)}\left(-\tau_{-}(x, v)\right)\right) & =\left.\frac{\partial}{\partial t}\right|_{t=0} f_{-}\left(\vec{\gamma}_{\left(\vec{\gamma}_{(x, v)}(t)\right)}\left(-\tau_{-}\left(\vec{\gamma}_{(x, v)}(t)\right)\right)\right) \\
& =\left.\frac{\partial}{\partial t}\right|_{t=0} f_{-}\left(\vec{\gamma}_{(x, v)}\left(-\tau_{-}(x, v)\right)\right)=0 .
\end{aligned}
$$

Proposition 2.5. If $\left\|\tau \sigma_{a}\right\|_{L^{\infty}}<\infty$ then $J: L^{1}\left(\Gamma_{-}, d \mu\right) \rightarrow \mathcal{W}$ with

$$
\left\|J f_{-}\right\|_{\mathcal{W}} \leq\left(1+\left\|\tau \sigma_{a}\right\|_{L^{\infty}}\right)\left\|f_{-}\right\|_{L^{1}\left(\Gamma_{-}, d \mu\right)} .
$$

The proof is carried out as in [CS2]. Set

$$
T_{0} f=-\mathcal{D} f-\sigma_{a} f, \quad T_{1} f(x, v)=\int_{T_{x} M} k\left(x, v^{\prime}, v\right) f\left(x, v^{\prime}\right) d v^{\prime},
$$

and put $T=T_{0}+T_{1}$. We wish to solve $T_{0} f+T_{1} f=0$ with $\left.f\right|_{\Gamma_{-}}=f_{-}$. To this end, multiply $T_{0} f=-T_{1} f$ by $E\left(x, v,-\tau_{-}(x, v), 0\right)$. Then

$$
\begin{aligned}
\mathcal{D}\left(E\left(x, v,-\tau_{-}(x, v), 0\right) f(x, v)\right) & =-E\left(x, v,-\tau_{-}(x, v), 0\right) T_{0} f(x, v) \\
& =E\left(x, v,-\tau_{-}(x, v), 0\right) T_{1} f(x, v)
\end{aligned}
$$

has solution

$$
\begin{aligned}
& E\left(x, v,-\tau_{-}(x, v), 0\right) f(x, v) \\
& \quad=\int_{0}^{\tau_{-}(x, v)} E\left(\vec{\gamma}_{(x, v)}\left(t-\tau_{-}(x, v)\right),-t, 0\right)\left(T_{1} f\right)\left(\vec{\gamma}_{(x, v)}\left(t-\tau_{-}(x, v)\right)\right) d t \\
& \quad=\int_{0}^{\tau_{-}(x, v)} E\left(x, v,-\tau_{-}(x, v), t-\tau_{-}(x, v)\right)\left(T_{1} f\right)\left(\vec{\gamma}_{(x, v)}\left(t-\tau_{-}(x, v)\right)\right) d t,
\end{aligned}
$$

where we have used the identities (6) and (7). Define $K$ by

$$
\begin{aligned}
& K f(x, v)=-E^{-1}\left(x, v,-\tau_{-}(x, v), 0\right) \int_{0}^{\tau_{-}(x, v)} E\left(x, v,-\tau_{-}(x, v), t-\tau_{-}(x, v)\right) \\
& \cdot\left(T_{1} f\right)\left(\vec{\gamma}_{(x, v)}\left(t-\tau_{-}(x, v)\right)\right) d t \\
&=-\int_{0}^{\tau_{-}(x, v)} E\left(x, v, 0, t-\tau_{-}(x, v)\right)\left(T_{1} f\right)\left(\vec{\gamma}_{(x, v)}\left(t-\tau_{-}(x, v)\right)\right) d t .
\end{aligned}
$$

Taking into account the boundary condition $\left.f\right|_{\Gamma_{-}}=f_{-}$,

$$
f(x, v)=-K f(x, v)+E\left(x, v, 0,-\tau_{-}(x, v)\right) f_{-}\left(\vec{\gamma}_{(x, v)}\left(-\tau_{-}(x, v)\right)\right),
$$

that is,

$$
(I+K) f=J f_{-} .
$$

Consider the unbounded operators $\mathbf{T}_{0} f=T_{0} f$ and $\mathbf{T} f=T f$ with domains

$$
\begin{aligned}
D\left(\mathbf{T}_{0}\right) & =\left\{f \in L^{1}(T M)\left|T_{0} f \in L^{1}(T M), f\right|_{\Gamma_{-}}=0\right\}, \\
D(\mathbf{T}) & =\left\{f \in L^{1}(T M)\left|T f \in L^{1}(T M), f\right|_{\Gamma_{-}}=0\right\} .
\end{aligned}
$$


Formally,

$$
\mathbf{T}_{0}^{-1} f(x, v)=-\int_{0}^{\tau_{-}(x, v)} E\left(x, v, 0, t-\tau_{-}(x, v)\right) f\left(\vec{\gamma}_{(x, v)}\left(t-\tau_{-}(x, v)\right)\right) d t
$$

and, again formally, $K=\mathbf{T}_{0}^{-1} T_{1}$. In the following proposition we show that on the appropriate spaces these formal statements are precise. Once again, the arguments mirror those in [CS2].

\section{Proposition 2.6.}

(i) The operators $\tau^{-1} \mathbf{T}_{0}^{-1}$ and $T_{1} \tau$ are bounded on $L^{1}(T M)$.

(ii) $K=\mathbf{T}_{0}^{-1} T_{1}$ is bounded on $L^{1}\left(T M, \tau^{-1} d v_{x} d \omega(x)\right)$, the operator norm of $K$ is bounded by $\left\|\tau \sigma_{p}\right\|_{L^{\infty}}<1$, and so $I+K$ is invertible on this space.

(iii) Equation (8) and hence (2) is uniquely solvable for $f_{-} \in L^{1}\left(\Gamma_{-}, d \mu\right)$ with solution $f \in \mathcal{W}$.

(iv) The albedo operator $\mathcal{A}: L^{1}\left(\Gamma_{-}, d \mu\right) \rightarrow L^{1}\left(\Gamma_{+}, d \mu\right)$ is a bounded map.

(v) The operator $\tau^{-1} \mathbf{T}^{-1}$ is bounded on $L^{1}(T M)$.

Proof. First, if $f \in L^{1}(T M)$ then

$$
\begin{aligned}
& \left\|\tau^{-1} \mathbf{T}_{0}^{-1} f\right\|_{L^{1}(T M)} \\
& \leq \int_{\Gamma_{-}} \int_{0}^{\tau_{+}\left(x^{\prime}, v^{\prime}\right)} \frac{1}{\tau}\left|\int_{0}^{\tau_{-}\left(\vec{\gamma}_{\left(x^{\prime}, v^{\prime}\right)}(t)\right)} f\left(\vec{\gamma}_{\left(\vec{\gamma}_{\left(x^{\prime}, v^{\prime}\right)}(t)\right)}\left(s-\tau_{-}\left(\vec{\gamma}_{\left(x^{\prime}, v^{\prime}\right)}(t)\right)\right)\right) d s\right| d t d \mu \\
& \leq \int_{\Gamma_{-}} \int_{0}^{\tau_{+}\left(x^{\prime}, v^{\prime}\right)} \frac{1}{\tau_{+}\left(x^{\prime}, v^{\prime}\right)} \int_{0}^{\tau_{+}\left(x^{\prime}, v^{\prime}\right)}\left|f\left(\vec{\gamma}_{\left(x^{\prime}, v^{\prime}\right)}(s)\right)\right| d s d t d \mu \\
& =\int_{\Gamma_{-}} \int_{0}^{\tau_{+}\left(x^{\prime}, v^{\prime}\right)}\left|f\left(\vec{\gamma}_{\left(x^{\prime}, v^{\prime}\right)}(s)\right)\right| d s d \mu=\|f\|_{L^{1}(T M) .}
\end{aligned}
$$

Next,

$$
\left\|T_{1} \tau f\right\|_{L^{1}(T M)} \leq\left\|\frac{1}{\tau} \sigma_{p}\right\|_{L^{\infty}(T M)}\|f\|_{L^{1}(T M)},
$$

by (4). It follows that $K=\mathbf{T}_{0}^{-1} T_{1}$ and

$$
\begin{aligned}
\left\|\tau^{-1} K f\right\|_{L^{1}(T M)} & =\left\|\tau^{-1} \mathbf{T}_{0}^{-1} T_{1} f\right\|_{L^{1}(T M)} \leq\left\|T_{1} f\right\|_{L^{1}(T M)} \\
& \leq\left\|\tau \sigma_{p}\right\|_{L^{\infty}(T M)}\left\|\tau^{-1} f\right\|_{L^{1}(T M)} \\
& <\left\|\tau^{-1} f\right\|_{L^{1}(T M)},
\end{aligned}
$$

by (4). Thus $(I+K)$ is invertible on $L^{1}\left(T M, \tau^{-1} d v_{x} d \omega(x)\right)$ and (8) has solution $f=(I+K)^{-1} J f_{-}$. We now show that $f \in \mathcal{W}$ and so has a 
well-defined trace. We have

$$
\begin{aligned}
\left\|\tau_{-} f\right\|_{L^{1}(T M)} & \leq\left(1-\left\|\tau \sigma_{p}\right\|_{L^{\infty}(T M)}\right)^{-1}\left\|\tau^{-1} J f_{-}\right\|_{L^{1}(T M)} \\
& \leq\left(1-\left\|\tau \sigma_{p}\right\|_{L^{\infty}(T M)}\right)^{-1}\left\|f_{-}\right\|_{L^{1}\left(\Gamma_{-}, d \mu\right)},
\end{aligned}
$$

where the last inequality follows from

$$
\begin{aligned}
\left\|\tau^{-1} J f\right\|_{L^{1}(T M)} & \leq \int_{M} \int_{T_{x} M} \tau^{-1}(x, v)\left|f_{-}\left(\vec{\gamma}_{(x, v)}\left(-\tau_{-}(x, v)\right)\right)\right| d v_{x} d \omega(x) \\
& =\int_{\Gamma_{-}} \int_{0}^{\tau_{+}\left(x^{\prime}, v^{\prime}\right)} \tau_{+}^{-1}\left(x^{\prime}, v^{\prime}\right)\left|f_{-}\left(x^{\prime}, v^{\prime}\right)\right| d t d \mu\left(x^{\prime}, v^{\prime}\right) \\
& =\left\|f_{-}\right\|_{L^{1}\left(\Gamma_{-}, d \mu\right)} .
\end{aligned}
$$

Since $T f=0$ and $\mathcal{D} f=-\sigma_{a} f+T_{1} f$, we have

$$
\|\mathcal{D} f\|_{L^{1}(T M)} \leq\left(\left\|\tau \sigma_{a}\right\|_{L^{\infty}(T M)}+\left\|\tau \sigma_{p}\right\|_{L^{\infty}(T M)}\right)\left\|\tau^{-1} f\right\|_{L^{1}(T M)}<\infty
$$

from the previous estimate. Thus $f \in \mathcal{W}$, and applying Theorem 2.3 we have $\left.f\right|_{\Gamma_{+}}=\mathcal{A} f_{-} \in L^{1}\left(\Gamma_{+}, d \mu\right)$ with

$$
\begin{aligned}
\|\mathcal{A} f\|_{L^{1}\left(\Gamma_{+}, d \mu\right)} & \leq\|\mathcal{D} f\|_{L^{1}(T M)}+\left\|\tau^{-1} f\right\|_{L^{1}(T M)} \\
& \leq\left(1+\left\|\tau \sigma_{a}\right\|_{L^{\infty}(T M)}+\left\|\tau \sigma_{p}\right\|_{L^{\infty}(T M)}\right)\left\|\tau^{-1} f\right\|_{L^{1}(T M)} \\
& \leq\left(1+\left\|\tau \sigma_{a}\right\|_{L^{\infty}}+\left\|\tau \sigma_{p}\right\|_{L^{\infty}}\right)\left(1-\left\|\tau \sigma_{p}\right\|_{L^{\infty}}\right)^{-1}\left\|f_{-}\right\|_{L^{1}\left(\Gamma_{-}, d \mu\right)} .
\end{aligned}
$$

Finally, (v) follows by setting

$$
\mathbf{T}^{-1}=(I+K)^{-1} \mathbf{T}_{0}^{-1}: L^{1}(T M) \rightarrow L^{1}\left(T M, \tau^{-1} d v_{x} d \omega(x)\right) .
$$

\section{Singular solutions and the kernel of $\mathcal{A}$}

We now solve (2) in the sense of distributions with a singular boundary condition:

$$
\begin{gathered}
-\mathcal{D} f(x, v)-\sigma_{a}(x, v) f(x, v)+\int_{T_{x} M} k\left(x, v^{\prime \prime}, v\right) f\left(x, v^{\prime \prime}\right) d v_{x}^{\prime \prime}=0, \\
\left.f\right|_{\Gamma_{-}}=\delta_{\{\hat{x}, \hat{v}\}}\left(x^{\prime}, v^{\prime}\right),
\end{gathered}
$$

with $(\hat{x}, \hat{v}) \in \Gamma_{-}$. Here, $\delta_{\{\hat{x}, \hat{v}\}}\left(x^{\prime}, v^{\prime}\right)$ is a distribution on $\Gamma_{-}$defined by

$$
\left(\delta_{\{\hat{x}, \hat{v}\}}, \varphi\right)=\int_{\Gamma_{-}} \delta_{\{\hat{x}, \hat{v}\}}\left(x^{\prime}, v^{\prime}\right) \varphi\left(x^{\prime}, v^{\prime}\right) d \mu\left(x^{\prime}, v^{\prime}\right)=\varphi(\hat{x}, \hat{v}) .
$$

Let $\varphi_{-} \in C_{0}^{\infty}\left(\Gamma_{-}\right)$and $\varphi$ be the solution to

$$
\begin{aligned}
&-\mathcal{D} \varphi(x, v)-\sigma_{a}(x, v) \varphi(x, v)+\int_{T_{x} M} k\left(x, v, v^{\prime}\right) \varphi\left(x, v^{\prime}\right) d v_{x}^{\prime}=0, \\
&\left.\varphi\right|_{\Gamma_{-}}=\varphi_{-} .
\end{aligned}
$$


Then

$$
\varphi=(I+K)^{-1} J \varphi_{-}=J \varphi_{-}-K J \varphi_{-}+\mathbf{T}^{-1} T_{1} K J \varphi_{-} .
$$

We shall analyze the three terms in this expression for $\varphi$, determining the distribution kernel of the solution operator $\varphi_{-} \mapsto \varphi$ of (13), which solves (12) in the sense of distributions. This is the content of the following three propositions:

Proposition 3.1. For the first term in (14) we have

$$
J \varphi_{-}(x, v)=\int_{\Gamma_{-}} f_{0}\left(x, v, x^{\prime}, v^{\prime}\right) \varphi_{-}\left(x^{\prime}, v^{\prime}\right) d \mu\left(x^{\prime}, v^{\prime}\right),
$$

with

$$
f_{0}\left(x, v, x^{\prime}, v^{\prime}\right)=\int_{0}^{\tau_{+}\left(x^{\prime}, v^{\prime}\right)} E\left(x, v, 0,-\tau_{-}(x, v)\right) \delta_{(x, v)}\left(\vec{\gamma}_{\left(x^{\prime}, v^{\prime}\right)}(t)\right) d t .
$$

Proof. If $\psi \in C_{0}^{\infty}(T M)$ then

$$
\begin{aligned}
\left(J \varphi_{-},\right. & \psi) \\
& =\int_{\Gamma_{-}} \int_{0}^{\tau_{+}\left(x^{\prime}, v^{\prime}\right)} J \varphi_{-}\left(\vec{\gamma}_{\left(x^{\prime}, v^{\prime}\right)}(t)\right) \psi\left(\vec{\gamma}_{\left(x^{\prime}, v^{\prime}\right)}(t)\right) d t d \mu\left(x^{\prime}, v^{\prime}\right) \\
& =\int_{\Gamma_{-}} \varphi_{-}\left(x^{\prime}, v^{\prime}\right) \int_{0}^{\tau_{+}\left(x^{\prime}, v^{\prime}\right)} E\left(\vec{\gamma}_{\left(x^{\prime}, v^{\prime}\right)}(t), 0,-t\right) \psi\left(\vec{\gamma}_{\left(x^{\prime}, v^{\prime}\right)}(t)\right) d t d \mu\left(x^{\prime}, v^{\prime}\right)
\end{aligned}
$$

(since $-\tau_{-}\left(\vec{\gamma}_{\left(x^{\prime}, v^{\prime}\right)}(t)\right)=-t$ ),

$$
\begin{aligned}
=\int_{M} \int_{T_{x} M} \int_{\Gamma_{-}} \varphi_{-}\left(x^{\prime}, v^{\prime}\right) \int_{0}^{\tau_{+}\left(x^{\prime}, v^{\prime}\right)} E\left(x, v, 0,-\tau_{-}(x, v)\right) \\
\cdot \delta_{(x, v)}\left(\vec{\gamma}_{\left(x^{\prime}, v^{\prime}\right)}(t)\right) d t d \mu\left(x^{\prime}, v^{\prime}\right) \psi(x, v) d v_{x} d \omega(x),
\end{aligned}
$$

where $\delta_{(\bar{x}, \bar{v})}$ is the distribution on $T M$ defined by

$$
\left(\delta_{(\bar{x}, \bar{v})}, \varphi\right)=\int_{M} \int_{T_{x} M} \delta_{(\bar{x}, \bar{v})}(x, v) \varphi(x, v) d v_{x} d \omega=\varphi(\bar{x}, \bar{v}) .
$$

Thus,

$$
J \varphi_{-}(x, v)=\int_{\Gamma_{-}} f_{0}\left(x, v, x^{\prime}, v^{\prime}\right) \varphi\left(x^{\prime}, v^{\prime}\right) d \mu\left(x^{\prime}, v^{\prime}\right),
$$

with $f_{0}$ as given in the statement of the proposition.

Due to our assumptions on the metric, the following parallel translation map is globally well-defined: given $(x, v) \in T M$ and $y \in M$ denote by $\mathcal{P}(v ; x, y): T_{x} M \rightarrow T_{y} M$ the parallel translation of $v$ along the (unique) geodesic joining $x$ and $y$. 
Proposition 3.2. For the second term in (14) we have

$$
K J \varphi_{-}(x, v)=\int_{\Gamma_{-}} f_{1}\left(x, v, x^{\prime}, v^{\prime}\right) \varphi_{-}\left(x^{\prime}, v^{\prime}\right) d \mu\left(x^{\prime}, v^{\prime}\right)
$$

with

$$
\begin{aligned}
& f_{1}\left(x, v, x^{\prime}, v^{\prime}\right) \\
&=\int_{0}^{\tau_{+}\left(x^{\prime}, v^{\prime}\right)} \int_{0}^{\tau_{-}(x, v)} E\left(x, v, 0, s-\tau_{-}(x, v)\right) E\left(x^{\prime}, v^{\prime}, 0, r\right) \\
& \cdot k\left(\vec{\gamma}_{\left(x^{\prime}, v^{\prime}\right)}(r), \mathcal{P}\left(\dot{\gamma}_{(x, v)}\left(s-\tau_{-}(x, v)\right) ; \gamma_{(x, v)}\left(s-\tau_{-}(x, v)\right), \gamma_{\left(x^{\prime}, v^{\prime}\right)}(r)\right)\right) \\
& \cdot \delta_{\left\{\gamma_{(x, v)}\left(s-\tau_{-}(x, v)\right)\right\}}\left(\gamma_{\left(x^{\prime}, v^{\prime}\right)}(r)\right) d s d r .
\end{aligned}
$$

Proof. Consider, for $\psi \in C_{0}^{\infty}(T M)$,

$$
\left(K J \varphi_{-}, \psi\right)=\int_{\Gamma_{-}} \int_{0}^{\tau_{+}\left(x^{\prime}, v^{\prime}\right)} K J \varphi_{-}\left(\vec{\gamma}_{\left(x^{\prime}, v^{\prime}\right)}(t)\right) \psi\left(\vec{\gamma}_{\left(x^{\prime}, v^{\prime}\right)}(t)\right) d t d \mu\left(x^{\prime}, v^{\prime}\right) .
$$

First, using identities (6) and (7) together with the fact that $\tau_{-}\left(x^{\prime}, v^{\prime}\right)=0$ when $\left(x^{\prime}, v^{\prime}\right) \in \Gamma_{-}$, we get

$$
\begin{aligned}
& K J \varphi_{-}\left(\vec{\gamma}_{\left(x^{\prime}, v^{\prime}\right)}(t)\right)=-\int_{0}^{t} E\left(x^{\prime}, v^{\prime}, t, s\right) T_{1} J \varphi_{-}\left(\vec{\gamma}_{\left(x^{\prime}, v^{\prime}\right)}(s)\right) d s \\
& T_{1} J \varphi_{-}\left(\vec{\gamma}_{\left(x^{\prime}, v^{\prime}\right)}(s)\right)= \int_{T_{\gamma_{\left(x^{\prime}, v^{\prime}\right)}(s)} M} k\left(\gamma_{\left(x^{\prime}, v^{\prime}\right)}(s), w, \dot{\gamma}_{\left(x^{\prime}, v^{\prime}\right)}(s)\right) \\
& \cdot J \varphi_{-}\left(\gamma_{\left(x^{\prime}, v^{\prime}\right)}(s), w\right) d w_{\gamma_{\left(x^{\prime}, v^{\prime}\right)}(s)}
\end{aligned}
$$

and

$$
\begin{aligned}
J \varphi_{-}\left(\gamma_{\left(x^{\prime}, v^{\prime}\right)}(s), w\right)=-E\left(\gamma_{\left(x^{\prime}, v^{\prime}\right)}(s), w,-\tau_{-}\left(\gamma_{\left(x^{\prime}, v^{\prime}\right)}(s), w\right), 0\right) \\
\cdot \varphi_{-}\left(\vec{\gamma}_{\left(\gamma_{\left(x^{\prime}, v^{\prime}\right)}(s), w\right)}\left(-\tau_{-}\left(\gamma_{\left(x^{\prime}, v^{\prime}\right)}(s), w\right)\right)\right) .
\end{aligned}
$$

Thus,

$$
\begin{aligned}
& \left(K J \varphi_{-}, \psi\right) \\
& =\int_{\Gamma_{-}} \int_{0}^{\tau_{+}\left(x^{\prime}, v^{\prime}\right)} \int_{0}^{t} \int_{T_{\gamma_{\left(x^{\prime}, v^{\prime}\right)}(s)} M} E\left(x^{\prime}, v^{\prime}, t, s\right) \\
& \quad \cdot E\left(\gamma_{\left(x^{\prime}, v^{\prime}\right)}(s), w,-\tau_{-}\left(\gamma_{\left(x^{\prime}, v^{\prime}\right)}(s), w\right), 0\right) k\left(\gamma_{\left(x^{\prime}, v^{\prime}\right)}(s), w, \dot{\gamma}_{\left(x^{\prime}, v^{\prime}\right)}(s)\right) \\
& \quad \cdot \varphi_{-}(\bar{x}, \bar{v}) \psi\left(\vec{\gamma}_{\left(x^{\prime}, v^{\prime}\right)}(t)\right) d w d s d t d \mu\left(x^{\prime}, v^{\prime}\right)
\end{aligned}
$$

where $(\bar{x}, \bar{v})=\left(\vec{\gamma}_{\left(\gamma_{\left(x^{\prime}, v^{\prime}\right)}(s), w\right)}\left(-\tau_{-}\left(\gamma_{\left(x^{\prime}, v^{\prime}\right)}(s), w\right)\right)\right)$; see Figure 1 on the next page.

We now perform the change of variables from $\left(x^{\prime}, v^{\prime}, t\right)$ to $(x, v)$, where $x=\gamma_{\left(x^{\prime}, v^{\prime}\right)}(t)$ and $v=\dot{\gamma}_{\left(x^{\prime}, v^{\prime}\right)}(t)$. Then $t=\tau_{-}(x, v)$,

$$
\gamma_{\left(x^{\prime}, v^{\prime}\right)}(s)=\gamma_{(x, v)}\left(s-\tau_{-}(x, v)\right), \quad \text { and } \quad \dot{\gamma}_{\left(x^{\prime}, v^{\prime}\right)}(s)=\dot{\gamma}_{(x, v)}\left(s-\tau_{-}(x, v)\right) \text {. }
$$




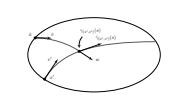

\section{Figure 1.}

Using parallel translation we may now introduce a delta distribution to obtain

$\left(K J \varphi_{-}, \psi\right)$

$$
\begin{aligned}
=\int_{M} \int_{T_{x} M} & \int_{0}^{\tau_{-}(x, v)} \int_{M} \delta_{\gamma_{(x, v)}\left(s-\tau_{-}(x, v)\right)}(y) \int_{T_{y} M} E\left(x, v, 0, s-\tau_{-}(x, v)\right) \\
& \cdot E\left(y, w,-\tau_{-}(y, w), 0\right) \\
& \cdot k\left(y, w, \mathcal{P}\left(\dot{\gamma}_{(x, v)}\left(s-\tau_{-}(x, v)\right) ; \gamma_{(x, v)}\left(s-\tau_{-}(x, v)\right), y\right)\right) \\
& \cdot \varphi_{-}\left(\vec{\gamma}_{(y, w)}\left(-\tau_{-}(y, w)\right)\right) \psi(x, v) d w_{y} d \omega(y) d s d v_{x} d \omega(x),
\end{aligned}
$$

where

$$
\left(\delta_{x}, \varphi\right)_{M}=\int_{M} \delta_{x}(y) \varphi(y) d \omega(y)=\varphi(x)
$$

Make another change of variables $\left(y^{\prime}, w^{\prime}, r\right)=\left(\vec{\gamma}_{(y, w)}\left(-\tau_{-}(y, w)\right), \tau_{-}(y, w)\right)$ and interchange the order of integration $d r d \mu\left(y^{\prime}, w^{\prime}\right) d s$ to $d s d r d \mu\left(y^{\prime}, w^{\prime}\right)$. We obtain

$$
\begin{aligned}
& \left(K J \varphi_{-}, \psi\right) \\
& =\int_{M} \int_{T_{x} M} \psi(x, v) \int_{\Gamma_{-}} \varphi_{-}\left(y^{\prime}, w^{\prime}\right) \int_{0}^{\tau_{+}\left(y^{\prime}, w^{\prime}\right)} \int_{0}^{\tau_{-}(x, v)} E\left(x, v, 0, s-\tau_{-}(x, v)\right) \\
& \quad \cdot E\left(y^{\prime}, w^{\prime}, 0, r\right) \\
& \quad \cdot k\left(\vec{\gamma}_{\left(y^{\prime}, w^{\prime}\right)}(r), \mathcal{P}\left(\dot{\gamma}_{(x, v)}\left(s-\tau_{-}(x, v)\right) ; \gamma_{(x, v)}\left(s-\tau_{-}(x, v)\right), \gamma_{\left(y^{\prime}, w^{\prime}\right)}(r)\right)\right) \\
& \quad \cdot \delta_{\left(\gamma_{(x, v)}\left(s-\tau_{-}(x, v)\right)\right)}\left(\gamma_{\left(y^{\prime}, w^{\prime}\right)}(r)\right) d s d r d \mu\left(y^{\prime}, w^{\prime}\right) d v_{x} d \omega(x) .
\end{aligned}
$$

Thus we have (16).

Proposition 3.3. For the third and final term in (14) we have

$$
\mathbf{T}^{-1} T_{1} K J \varphi_{-}(x, v)=\int_{\Gamma_{-}} f_{2}\left(x, v, x^{\prime}, v^{\prime}\right) \varphi_{-}\left(x^{\prime}, v^{\prime}\right) d \mu\left(x^{\prime}, v^{\prime}\right)
$$

with $f_{2} \in L^{\infty}\left(\Gamma_{-} ; \mathcal{W}\right)$. 
Proof. We have

$$
\begin{aligned}
& T_{1} K J \varphi_{-}(x, v) \\
& =\int_{T_{x} M} \int_{0}^{\tau_{-}\left(x, w^{\prime}\right)} \int_{T_{\hat{x}} M} k\left(x, w^{\prime}, v\right) k\left(\hat{x}, w, \dot{\gamma}_{\left(x, w^{\prime}\right)}\left(t-\tau_{-}\left(x, w^{\prime}\right)\right)\right) \\
& \cdot E\left(x, w^{\prime}, 0, t-\tau_{-}\left(x, w^{\prime}\right)\right) E\left(\hat{x}, w,-\tau_{-}(\hat{x}, w), 0\right) \\
& \cdot \varphi_{-}\left(\vec{\gamma}_{(\hat{x}, w)}\left(-\tau_{-}(\hat{x}, w)\right)\right) d w_{\hat{x}} d t d w_{x}^{\prime}
\end{aligned}
$$

where $\hat{x}=\gamma_{\left(x, w^{\prime}\right)}\left(t-\tau_{-}\left(x, w^{\prime}\right)\right)$. Take the $L^{1}(T M)$ norm of $T_{1} K J \varphi_{-}(x, v)$. We observe that

(18) $\left\|T_{1} K J \varphi_{-}(x, v)\right\|_{L^{1}(T M)}$

$$
\begin{aligned}
& \leq\left\|T_{1} \tau\right\|_{L^{1}(T M) \rightarrow L^{1}(T M)}\left\|\tau^{-1} K \tau\right\|_{L^{1}(T M) \rightarrow L^{1}(T M)}\left\|\tau^{-1} J \varphi_{-}\right\|_{L^{1}(T M)} \\
& \leq\left\|\varphi_{-}\right\|_{L^{1}\left(\Gamma_{-}, d \mu\right)},
\end{aligned}
$$

by (9), (4), (10) and (11). Thus by Fubini's theorem, the integral above defining $T_{1} K J \varphi_{-}(x, v)$ is absolutely convergent for a.e. $(x, v) \in T M$.

In the distributional sense, we make the following computation of the distribution kernel of $T_{1} K J$ : for $x \in M$ let $\mathcal{S}(x)=\left\{\hat{v} \in T_{x} M \mid\|\hat{v}\|=1\right\}$ and extend all functions defined on $T_{x} M$ by zero to be defined on all of $\{r \hat{v} \mid r \geq 0, \hat{v} \in \mathcal{S}(x)\}$. Let $\left(x, v, x^{\prime}, v^{\prime}\right) \in \Gamma_{+} \times \Gamma_{-}$. Then, as in (17),

$$
\begin{gathered}
\left(T_{1} K J \delta_{\left(x^{\prime}, v^{\prime}\right)}\right)(x, v) \\
=\int_{T_{x} M} \int_{0}^{\tau_{-}\left(x, w^{\prime}\right)} \int_{T_{\hat{x}} M} k\left(x, w^{\prime}, v\right) k\left(\hat{x}, w, \dot{\gamma}_{\left(x, w^{\prime}\right)}\left(t-\tau_{-}\left(x, w^{\prime}\right)\right)\right) \\
\cdot E\left(x, w^{\prime}, 0, t-\tau_{-}\left(x, w^{\prime}\right)\right) E\left(\hat{x}, w,-\tau_{-}(\hat{x}, w), 0\right) \\
\cdot \delta_{\left(x^{\prime}, v^{\prime}\right)}\left(\vec{\gamma}_{(\hat{x}, w)}\left(-\tau_{-}(\hat{x}, w)\right)\right) d w_{\hat{x}} d t d w_{x}^{\prime} .
\end{gathered}
$$

We shall cease to write out the arguments of the functions $E$ in order to compress the presentation. Change the integration over $w^{\prime} \in T_{x} M$ to polar coordinates $\left(r, \hat{w}^{\prime}\right) \in \mathbb{R}^{+} \times \mathcal{S}(x)$ and make the change of variables $y=\hat{x}=$ $\gamma_{\left(x, \hat{w}^{\prime}\right)}\left(t-\tau_{-}\left(x, \hat{w}^{\prime}\right)\right)$. Due to the assumptions on the metric $g$ this is a global diffeomorphism. Let $J$ be the determinant of the Jacobian of this change of variables. If $\gamma$ is the unit speed geodesic joining $\gamma(0)=y$ to $\gamma(d)=x$ (for $d>0$ ), denote by $\hat{w}_{x}(y)=\dot{\gamma}(d) \in T_{x} M$ the (unit) tangent vector of $\gamma$ at $x$ and denote by $\hat{w}(y)=\dot{\gamma}(0) \in T_{y} M$ the (unit) tangent vector of $\gamma$ at $y$. Then

$$
\begin{aligned}
\left(T_{1} K J \delta_{\left(x^{\prime}, v^{\prime}\right)}\right)(x, v) & \\
= & \int_{0}^{\infty} \int_{M} \int_{V(y)} k\left(x, r \hat{w}_{x}(y), v\right) k(y, w, r \hat{w}(y)) \\
& \cdot E(\cdot) E(\cdot) \delta_{\left(x^{\prime}, v^{\prime}\right)}\left(\vec{\gamma}_{(y, w)}\left(-\tau_{-}(y, w)\right)\right)|J| d w_{y} d \omega(y) r^{n-2} d r .
\end{aligned}
$$


Change variables again setting $(y, w)=\vec{\gamma}_{\left(a^{\prime}, b^{\prime}\right)}(s)$ and integrate with respect to $d \mu\left(a^{\prime}, b^{\prime}\right)$ :

$$
\begin{array}{r}
\left(T_{1} K J \delta_{\left(x^{\prime}, v^{\prime}\right)}\right)(x, v) \\
=\int_{0}^{\infty} \int_{\Gamma_{-}} \int_{0}^{\tau_{+}\left(a^{\prime}, b^{\prime}\right)} k\left(x, r \hat{w}_{x}\left(\gamma_{\left(a^{\prime}, b^{\prime}\right)}(s)\right), v\right) k\left(\vec{\gamma}_{\left(a^{\prime}, b^{\prime}\right)}(s), r \hat{w}\left(\gamma_{\left(a^{\prime}, b^{\prime}\right)}(s)\right)\right) \\
\cdot E(\cdot) E(\cdot) \delta_{\left(x^{\prime}, v^{\prime}\right)}\left(a^{\prime}, b^{\prime}\right)|J| d s d \mu\left(a^{\prime}, b^{\prime}\right) r^{n-2} d r \\
=\int_{0}^{\infty} \int_{0}^{\tau_{+}\left(x^{\prime}, v^{\prime}\right)} k\left(x, r \hat{w}_{x}\left(\gamma_{\left(x^{\prime}, v^{\prime}\right)}(s)\right), v\right) k\left(\vec{\gamma}_{\left(x^{\prime}, v^{\prime}\right)}(s), r \hat{w}\left(\gamma_{\left(x^{\prime}, v^{\prime}\right)}(s)\right)\right) \\
\cdot E(\cdot) E(\cdot)|J| d s r^{n-2} d r .
\end{array}
$$

Claim 3.4. If $\varphi_{-} \in L^{1}\left(\Gamma_{-}, d \mu\right)$ then for $(x, v) \in T M$

$$
\left(T_{1} K J \varphi_{-}\right)(x, v)=\int_{\Gamma_{-}}\left(T_{1} K J \delta_{\left(x^{\prime}, v^{\prime}\right)}\right)(x, v) \varphi_{-}\left(x^{\prime}, v^{\prime}\right) d \mu\left(x^{\prime}, v^{\prime}\right) .
$$

Proof. By the definition of $T_{1} K J$ (see (17)), and manipulating the integral as above, we obtain

$$
\begin{array}{r}
\left(T_{1} K J \varphi_{-}\right)(x, v) \\
=\int_{T_{x} M} \int_{0}^{\tau_{-}\left(x, w^{\prime}\right)} \int_{T_{\hat{x}} M} k\left(x, w^{\prime}, v\right) k\left(\hat{x}, w, \dot{\gamma}_{\left(x, w^{\prime}\right)}\left(t-\tau_{-}\left(x, w^{\prime}\right)\right)\right) \\
\cdot E(\cdot) E(\cdot) \delta_{\left(x^{\prime}, v^{\prime}\right)}\left(\vec{\gamma}_{(\hat{x}, w)}\left(-\tau_{-}(\hat{x}, w)\right)\right) d w_{\hat{x}} d t d w_{x}^{\prime} \\
=\int_{0}^{\infty} \int_{\Gamma_{-}} \int_{0}^{\tau_{+}\left(a^{\prime}, b^{\prime}\right)} k\left(x, r \hat{w}_{x}\left(\gamma_{\left(a^{\prime}, b^{\prime}\right)}(s)\right), v\right) k\left(\vec{\gamma}_{\left(a^{\prime}, b^{\prime}\right)}(s), r \hat{w}\left(\gamma_{\left(a^{\prime}, b^{\prime}\right)}(s)\right)\right) \\
\cdot E(\cdot) E(\cdot) \varphi_{-}\left(a^{\prime}, b^{\prime}\right)|J| d s d \mu\left(a^{\prime}, b^{\prime}\right) r^{n-2} d r .
\end{array}
$$

Relabeling $\left(a^{\prime}, b^{\prime}\right)$ as $\left(x^{\prime}, v^{\prime}\right)$ and interchanging the order of integration from $d s d \mu\left(x^{\prime}, v^{\prime}\right) d r$ to $d s d r d \mu\left(x^{\prime}, v^{\prime}\right)$ we obtain the right-hand side of (19) as claimed.

From (18) we see that $T_{1} K J \varphi_{-} \in L^{1}(T M)$ for all $\varphi_{-} \in L^{1}\left(\Gamma_{-}, d \mu\right)$. Thus from (19) and the Riesz representation theorem for $L^{1}(T M)$-valued functionals, the kernel $T_{1} K J \delta_{\left(x^{\prime}, v^{\prime}\right)}(x, v)$ is in $L^{\infty}\left(\Gamma_{-}\left(x^{\prime}, v^{\prime}\right) ; L^{1}(T M)\right)$.

Define $f_{2}\left(\cdot, \cdot, x^{\prime}, v^{\prime}\right)=\mathbf{T}^{-1} T_{1} K J \delta_{\left(x^{\prime}, v^{\prime}\right)}$, where $\mathbf{T}^{-1}$ acts on the variables $(x, v)$ with $\left(x^{\prime}, v^{\prime}\right)$ as parameters. Then

$$
\mathbf{T}^{-1} T_{1} K J \varphi_{-}(x, v)=\int_{\Gamma_{-}} f_{2}\left(x, v, x^{\prime}, v^{\prime}\right) \varphi_{-}\left(x^{\prime}, v^{\prime}\right) d \mu\left(x^{\prime}, v^{\prime}\right) .
$$

(The interchange of the operator $\mathbf{T}^{-1}$ and the integration is justified by the continuity of $\mathbf{T}^{-1}$; see [DU] for example.) We show that $f_{2} \in L^{\infty}\left(\Gamma_{-} ; \mathcal{W}\right)$, so that restriction to $(x, v) \in \Gamma_{+}$is well-defined. By Proposition $2.6, \mathbf{T}^{-1}$ is 
bounded from $L^{1}(T M)$ to $L^{1}\left(T M, \tau^{-1} d v_{x} d \omega(x)\right)$, so

$$
f_{2} \in L^{\infty}\left(\Gamma_{-} ; L^{1}\left(T M, \tau^{-1} d v_{x} d \omega(x)\right)\right) .
$$

Next, $\mathcal{D} f_{2}=-\sigma_{a} f_{2}+T_{1} f_{2}+T_{1} K J \delta_{\left(x^{\prime}, v^{\prime}\right)}$ and $f_{2} \mapsto-\sigma_{a} f_{2}+T_{1} f_{2}$ is bounded from $L^{1}\left(T M, \tau^{-1} d v_{x} d \omega(x)\right)$ to $L^{1}(T M)$ by (3) and (4), so

$$
\mathcal{D} f_{2} \in L^{\infty}\left(\Gamma_{-} ; L^{1}(T M)\right) \text {. }
$$

To summarize our analysis of the terms in (14), we have shown that (13) has solution

$$
\varphi=\int_{\Gamma_{-}} f\left(x, v, x^{\prime}, v^{\prime}\right) \varphi_{-}\left(x^{\prime}, v^{\prime}\right) d \mu\left(x^{\prime}, v^{\prime}\right)
$$

where the integral is in distributional sense and $f$ is the sum of the three terms given by (15), (16) and $f_{2}$ above. Furthermore, $f$ solves (12) in the sense of distributions. In other words, $f$ is the distribution kernel of the solution operator $\varphi_{-} \mapsto \varphi$ of (13). We proceed now to show that the distribution kernel $\alpha\left(x, v, x^{\prime}, v^{\prime}\right)$ (where $\left.(x, v) \in \Gamma_{+},\left(x^{\prime}, v^{\prime}\right) \in \Gamma_{-}\right)$of the albedo operator $\mathcal{A}$ is the restriction of $f$ to $(x, v) \in \Gamma_{+}$.

Theorem 3.5. The distribution kernel $\alpha\left(x, v, x^{\prime}, v^{\prime}\right)$ of $\mathcal{A}$ is expressible as a sum $\alpha=\alpha_{0}+\alpha_{1}+\alpha_{2}$, with

$$
\begin{aligned}
& \alpha_{0}= E\left(x, v,-\tau_{-}(x, v), 0\right) \delta_{\left\{\vec{\gamma}_{(x, v)}\left(-\tau_{-}(x, v)\right)\right\}}\left(x^{\prime}, v^{\prime}\right) \\
&= \exp \left(\int_{-\tau_{-}(x, v)}^{0} \sigma_{a}\left(\vec{\gamma}_{(x, v)}(r)\right) d r\right) \delta_{\left\{\vec{\gamma}_{(x, v)}\left(-\tau_{-}(x, v)\right)\right\}}\left(x^{\prime}, v^{\prime}\right), \\
& \alpha_{1}=\int_{0}^{\tau_{+}\left(x^{\prime}, v^{\prime}\right)} \int_{0}^{\tau_{-}(x, v)} E\left(x, v, 0, s-\tau_{-}(x, v)\right) E\left(x^{\prime}, v^{\prime}, 0, r\right) \\
& \quad \cdot k\left(\vec{\gamma}_{\left(x^{\prime}, v^{\prime}\right)}(r), \mathcal{P}\left(\dot{\gamma}_{(x, v)}\left(s-\tau_{-}(x, v)\right) ; \gamma_{(x, v)}\left(s-\tau_{-}(x, v)\right), \gamma_{\left(x^{\prime}, v^{\prime}\right)}(r)\right)\right) \\
& \quad \cdot \delta_{\left\{\gamma_{(x, v)}\left(s-\tau_{-}(x, v)\right)\right\}}\left(\gamma_{\left(x^{\prime}, v^{\prime}\right)}(r)\right) d s d r,
\end{aligned}
$$

$\alpha_{2} \in L^{\infty}\left(\Gamma_{-} ; L^{1}\left(\Gamma_{+}, d \mu\right)\right)$.

Proof. We have formally $\alpha_{j}=f_{j}$ restricted to $(x, v) \in \Gamma_{+}, j=0,1,2$. Let $\varphi_{-} \in C_{0}^{m}\left(\Gamma_{-}\right)$and for $(x, v) \in \Gamma_{+}$consider $\varphi_{j}(x, v)=\int_{\Gamma_{-}} f_{j} \varphi_{-} d \mu\left(x^{\prime}, v^{\prime}\right)$. From (15), changing variables to $(y, w)=\vec{\gamma}_{\left(x^{\prime}, v^{\prime}\right)}(t)$, we have

$$
\begin{aligned}
\varphi_{0} & (x, v) \\
& =\int_{M} \int_{T_{y} M} E\left(x, v,-\tau_{-}(x, v), 0\right) \delta_{\{x, v\}}(y, w) \varphi_{-}\left(\vec{\gamma}_{(y, w)}\left(-\tau_{-}(y, w)\right)\right) d w_{y} d \omega \\
& =E\left(x, v,-\tau_{-}(x, v), 0\right) \varphi_{-}\left(\vec{\gamma}_{(x, v)}\left(-\tau_{-}(x, v)\right)\right) \\
& =\int_{\Gamma_{-}} \alpha_{0}\left(x, v, x^{\prime}, v^{\prime}\right) \varphi_{-}\left(x^{\prime}, v^{\prime}\right) d \mu\left(x^{\prime}, v^{\prime}\right),
\end{aligned}
$$


which justifies the expression for $\alpha_{0}$. The expression for $\alpha_{1}$ is nothing more than a restatement of (16) with $(x, v) \in \Gamma_{+}$. That $\alpha_{2} \in L^{\infty}\left(\Gamma_{-} ; L^{1}\left(\Gamma_{+}, d \mu\right)\right)$ follows from (20), (21) and Proposition 2.5.

\section{The solution to the inverse problem}

In this section we show that from the distribution kernel $\alpha$ of the albedo operator $\mathcal{A}$ we can isolate the terms that differ in strength of singularity. In dimensions 3 and higher, we isolate $\alpha_{0}$ and $\alpha_{1}$ and show that this yields the absorption coefficient $\sigma_{a}$ and the scattering kernel $k$. We are able to do so because $\alpha_{0}$ and $\alpha_{1}$ are delta-type singularities in $\Gamma_{+} \times \Gamma_{-}$supported on varieties of differing dimension and $\alpha_{2}$ is an $L^{\infty}$ function. In dimension 2 , we isolate $\alpha_{0}$ from $\alpha$, which yields $\sigma_{a}$, but are unable to determine $k$ since in this case $\alpha_{1}$ is in fact a locally $L^{1}$ function and so not distinguishable from $\alpha_{2}$.

We shall use the following global coordinates on $T M$ : fix $p \in M$ and let $\left\{E_{i}\right\}$ be an orthonormal basis for $T_{p} M$. Define $E_{p}: \mathbb{R}^{n} \rightarrow T_{p} M$ by $E_{p}\left(x^{1}, \ldots, x^{n}\right)=\sum x^{i} E_{i}$. Let $\operatorname{Exp}_{p}: T_{p} M \rightarrow M$ denote the exponential map based at $p$. Note that $\operatorname{Exp}_{p}$ provides a diffeomorphism from $\operatorname{Exp}_{p}^{-1}(M)$ to $M$ due to our assumptions on the metric. Recall that $\mathcal{P}(v ; x, p): T_{x} M \rightarrow$ $T_{p} M$ is the parallel translation of $v$ along the unique geodesic joining $x$ and $p$. We may now define global coordinates for $T M$ by $\phi: T M \rightarrow \mathbb{R}^{n} \times \mathbb{R}^{n}$,

$$
\phi(x, v)=\left(\phi_{1}(x), \phi_{2}(v)\right)=\left(\left(E_{p}^{-1} \circ \operatorname{Exp}_{p}^{-1}\right)(x),\left(E_{p}^{-1} \circ \mathcal{P}\right)(v ; x, p)\right) .
$$

We first show that one can isolate $\alpha_{0}$ from $\alpha$. Let $\psi \in C_{0}^{\infty}(\mathbb{R})$ satisfy $0 \leq \psi \leq 1, \psi(0)=1$, and $\int \psi(x) d x=1$. Define $\psi_{\varepsilon}(x)=\psi(x / \varepsilon)$. Now let $\varphi: \Gamma_{+} \times \Gamma_{-} \rightarrow \mathbb{R}$ be a defining function for the support of the distribution $\delta_{\left\{\vec{\gamma}_{(x, v)}\left(-\tau_{-}(x, v)\right)\right\}}\left(x^{\prime}, v^{\prime}\right)$; that is, set

$$
\begin{aligned}
& \varphi\left(x, v, x^{\prime}, v^{\prime}\right) \\
& \quad=\left\|\phi_{1}\left(x^{\prime}\right)-\phi_{1}\left(\gamma_{(x, v)}\left(-\tau_{-}(x, v)\right)\right)\right\|^{2}+\left\|\phi_{2}\left(v^{\prime}\right)-\phi_{2}\left(\dot{\gamma}_{(x, v)}\left(-\tau_{-}(x, v)\right)\right)\right\|^{2},
\end{aligned}
$$

where the norm $\|\cdot\|$ is the Euclidean norm in $\mathbb{R}^{n}$. We have that the support of $\delta_{\left\{\vec{\gamma}_{(x, v)}\left(-\tau_{-}(x, v)\right)\right\}}\left(x^{\prime}, v^{\prime}\right)$ is the set $\left\{\varphi\left(x, v, x^{\prime}, v^{\prime}\right)=0\right\}$.

Proposition 4.1. The following limit holds in $L_{\mathrm{loc}}^{1}\left(\Gamma_{+}, d \mu(x, v)\right)$ :

$$
\begin{aligned}
\lim _{\varepsilon \rightarrow 0} \int_{\Gamma_{-}} \alpha\left(x, v, x^{\prime}, v^{\prime}\right)\left(\psi_{\varepsilon} \circ \varphi\right)\left(x, v, x^{\prime}, v^{\prime}\right) & d \mu\left(x^{\prime}, v^{\prime}\right) \\
& =\exp \left(\int_{-\tau_{-}(x, v)}^{0} \sigma_{a}\left(\vec{\gamma}_{(x, v)}(r)\right) d r\right)
\end{aligned}
$$

where the integral is meant in the sense of distributions.

Proof. When $\alpha$ is replaced by $\alpha_{0}$ the result is immediate. It remains to show that when $\alpha$ is replaced by $\alpha_{1}$ and $\alpha_{2}$ the limit vanishes. Consider first $\alpha_{1}$. 
We shall show that the limit vanishes when considered in

$$
L^{1}\left(\left\{(x, v) \in \Gamma_{+} \mid\|v\|_{g_{x}} \leq M\right\}, d \mu(x, v)\right),
$$

for any $M>0$. Let $0 \leq \chi \in C_{0}^{\infty}(\mathbb{R})$. Then

$$
\begin{aligned}
0 \leq & \int_{\Gamma_{+}} \int_{\Gamma_{-}} \chi\left(\|v\|_{g_{x}}\right) \alpha_{1}\left(x, v, x^{\prime}, v^{\prime}\right)\left(\psi_{\varepsilon} \circ \varphi\right)\left(x, v, x^{\prime}, v^{\prime}\right) d \mu\left(x^{\prime}, v^{\prime}\right) d \mu(x, v) \\
\leq & \int_{\Gamma_{+}} \int_{\Gamma_{-}} \int_{0}^{\tau_{+}\left(x^{\prime}, v^{\prime}\right)} \int_{0}^{\tau_{-}(x, v)} \chi\left(\|v\|_{g_{x}}\right) \\
& \cdot k\left(\vec{\gamma}_{\left(x^{\prime}, v^{\prime}\right)}(r), \mathcal{P}\left(\dot{\gamma}_{(x, v)}\left(s-\tau_{-}(x, v)\right) ; \gamma_{(x, v)}\left(s-\tau_{-}(x, v)\right), \gamma_{\left(x^{\prime}, v^{\prime}\right)}(r)\right)\right) \\
& \cdot \delta_{\left\{\gamma_{(x, v)}\left(s-\tau_{-}(x, v)\right)\right\}}\left(\gamma_{\left(x^{\prime}, v^{\prime}\right)}(r)\right)\left(\psi_{\varepsilon} \circ \varphi\right)\left(x, v, x^{\prime}, v^{\prime}\right) \\
& \cdot d s d r d \mu\left(x^{\prime}, v^{\prime}\right) d \mu(x, v) \\
\leq \int_{M} \int_{T_{y} M} \int_{T_{y} M} \chi\left(\|w\|_{g_{y}}\right) k\left(y, w^{\prime}, w\right) & \cdot\left(\psi_{\varepsilon} \circ \varphi\right)\left(\vec{\gamma}_{(y, w)}\left(\tau_{+}(y, w)\right), \vec{\gamma}_{\left(y, w^{\prime}\right)}\left(-\tau_{-}\left(y, w^{\prime}\right)\right)\right) d w_{y}^{\prime} d w_{y} d \omega(y),
\end{aligned}
$$

where $\left(y^{\prime}, w^{\prime}\right)=\vec{\gamma}_{\left(x^{\prime}, v^{\prime}\right)}(r),(y, w)=\vec{\gamma}_{(x, v)}\left(s-\tau_{-}(x, v)\right)$ and we have used the fact that $\|w\|_{g_{y}}=\left\|\dot{\gamma}_{(x, v)}\left(s-\tau_{-}(x, v)\right)\right\|_{g_{y}}=\|v\|_{g_{x}}$.

Now

$$
\begin{aligned}
&\left(\psi_{\varepsilon} \circ \varphi\right)\left(\vec{\gamma}_{(y, w)}\left(\tau_{+}(y, w)\right), \vec{\gamma}_{\left(y, w^{\prime}\right)}\left(-t_{-}\left(y, w^{\prime}\right)\right)\right) \\
&=\psi_{\varepsilon}\left(\left\|\phi_{1}\left(\gamma_{\left(y, w^{\prime}\right)}\left(-\tau_{-}\left(y, w^{\prime}\right)\right)\right)-\phi_{1}\left(\gamma_{(y, w)}\left(-\tau_{-}(y, w)\right)\right)\right\|^{2}\right.\left.+\left\|\phi_{2}\left(\dot{\gamma}_{\left(y, w^{\prime}\right)}\left(-\tau_{-}\left(y, w^{\prime}\right)\right)\right)-\phi_{2}\left(\dot{\gamma}_{(y, w)}\left(-\tau_{-}(y, w)\right)\right)\right\|^{2}\right) \\
&=\psi_{\varepsilon}\left(\left\|\phi_{1}\left(\gamma_{\left(y, w^{\prime}\right)}\left(-\tau_{-}\left(y, w^{\prime}\right)\right)\right)-\phi_{1}\left(\gamma_{(y, w)}\left(-\tau_{-}(y, w)\right)\right)\right\|^{2}\right. \\
&\left.+\left\|\phi_{2}\left(w^{\prime}\right)-\phi_{2}(w)\right\|^{2}\right),
\end{aligned}
$$

so it follows that there is $C$ depending on $\psi$ such that

$$
\begin{aligned}
\operatorname{supp}\left(\psi_{\varepsilon} \circ \varphi\right)\left(\vec{\gamma}_{(y, w)}\left(\tau_{+}(y, w)\right), \vec{\gamma}_{\left(y, w^{\prime}\right)}\left(-t_{-}\left(y, w^{\prime}\right)\right)\right) & \\
& \subset\left\{\left(y, w^{\prime}, w\right) \in M \times T_{y} M \times T_{y} M \mid\left\|w^{\prime}-w\right\|_{g_{y}}<C \varepsilon\right\} .
\end{aligned}
$$

If we set $W_{\varepsilon}=\left\{\left(y, w^{\prime}, w\right) \mid\left\|w^{\prime}-w\right\|_{g_{y}}<C \varepsilon\right.$ and $\left.\|w\|_{g_{y}} \in \operatorname{supp} \chi\right\}$, then from (23) we have

$$
\begin{aligned}
0 & \leq \int_{\Gamma_{+}} \int_{\Gamma_{-}} \chi\left(\|v\|_{g_{x}}\right) \alpha_{1}\left(x, v, x^{\prime}, v^{\prime}\right)\left(\psi_{\varepsilon} \circ \varphi\right)\left(x, v, x^{\prime}, v^{\prime}\right) d \mu\left(x^{\prime}, v^{\prime}\right) d \mu(x, v) \\
& \leq \int_{W_{\varepsilon}} \chi\left(\|v\|_{g_{x}}\right) k\left(y, w^{\prime}, w\right) d w_{y}^{\prime} d w_{y} d \omega(y)
\end{aligned}
$$

tending to 0 as $\lambda \rightarrow 0$, since $\chi\left(\|v\|_{g_{x}}\right) k\left(y, w^{\prime}, w\right) \in L^{1}\left(M \times T_{y} M \times T_{y} M\right)$ and the measure of $W_{\varepsilon} \rightarrow 0$ as $\varepsilon \rightarrow 0$. 
Finally, for $\alpha_{2}$,

$$
\begin{aligned}
0 & \leq \int_{\Gamma_{+}}\left|\int_{\Gamma_{-}} \chi\left(\|v\|_{g_{x}}\right) \alpha_{2}\left(x, v, x^{\prime}, v^{\prime}\right)\left(\psi_{\varepsilon} \circ \varphi\right)\left(x, v, x^{\prime}, v^{\prime}\right) d \mu\left(x^{\prime}, v^{\prime}\right)\right| d \mu(x, v) \\
& \leq \int_{V_{\varepsilon}} \chi\left(\|v\|_{g_{x}}\right)\left|\alpha_{2}\left(x, v, x^{\prime}, v^{\prime}\right)\right| d \mu\left(x^{\prime}, v^{\prime}\right) d \mu(x, v),
\end{aligned}
$$

tending to 0 as $\lambda \rightarrow 0$. Here

$\operatorname{supp}\left(\psi_{\varepsilon} \circ \varphi\right)\left(x, v, x^{\prime}, v^{\prime}\right)$

$$
\begin{array}{r}
\subset V_{\varepsilon}=\left\{\left(x, v, x^{\prime}, v^{\prime}\right) \in \Gamma_{+} \times \Gamma_{-} \mid\left\|\mathcal{P}\left(v^{\prime} ; x^{\prime}, p\right)-\mathcal{P}(v ; x, p)\right\|_{g_{p}}<C \varepsilon\right. \\
\text { and } \left.\left\|v^{\prime}\right\|_{g_{x^{\prime}}},\|v\|_{g_{x}} \in \operatorname{supp} \chi\right\}
\end{array}
$$

and the limit holds as stated since Theorem 3.5 gives

$$
\chi\left(\|v\|_{g_{x}}\right) \alpha_{2}\left(x, v, x^{\prime}, v^{\prime}\right) \in L^{1}\left(\Gamma_{+} \times \Gamma_{-}, d \mu(x, v) d \mu\left(x^{\prime}, v^{\prime}\right)\right)
$$

and the measure of $V_{\varepsilon}$ tends to 0 as $\varepsilon \rightarrow 0$.

Theorem 4.2. Suppose $M \subset \mathbb{R}^{n}, n \geq 2$, is a bounded domain with $C^{\infty}$ boundary, that $g$ is a simple Riemannian metric on $M$, and that assumptions (3) and (4) hold. Then from the kernel of $\mathcal{A}$ we may determine the absorption coefficient $\sigma_{a}(x, v)=\sigma_{a}\left(x,\|v\|_{g_{x}}\right)$.

Proof. From $\mathcal{A}$ we of course know $\alpha$; taking the limit as in Proposition 4.1 we obtain

$$
\int_{-\tau_{-}(x, v)}^{0} \sigma_{a}\left(\vec{\gamma}_{(x, v)}(r)\right) d r=\int_{-\tau_{-}(x, v)}^{0} \sigma_{a}\left(\gamma_{(x, v)}(r),\left\|v_{0}\right\|\right) d r
$$

for all $(x, v) \in \Gamma_{+}$, where $\left\|v_{0}\right\|=\left\|\dot{\gamma}_{(x, v)}(0)\right\|\left(=\left\|\dot{\gamma}_{(x, v)}(t)\right\|\right.$ for all $\left.t\right)$. That is, we know the integrals of $\sigma_{a}$ along the geodesics joining $\left(x^{\prime}, v^{\prime}\right)=$ $\vec{\gamma}_{(x, v)}\left(-\tau_{-}(x, v)\right) \in \Gamma_{-}$and $(x, v) \in \Gamma_{+}$. This is the geodesic ray transform of the function $\sigma_{a}$ and this transform is invertible. See [Sh1].

We work now towards determining $k$ by isolating $\alpha_{1}$. Fix $\left(y, w, w^{\prime}\right) \in$ $M \times T_{y} M \times T_{y} M$ with $w$ and $w^{\prime}$ linearly independent and let

$$
Z=\left\{\gamma_{(y, \bar{w})}\left(-\tau_{-}(y, \bar{w})\right) \mid \bar{w} \in \operatorname{span}\left\{w, w^{\prime}\right\}\right\} \subset \partial M .
$$

We let $h_{1}$ be a defining function for the set $Z$ as follows: for $z \in Z$ let $\gamma_{z}(t)$ be the geodesic joining $z$ to $y$ such that $\gamma_{z}(0)=z$ and $\gamma_{z}(1)=y$, and define $\pi\left(\dot{\gamma}_{z}(1)\right) \in T_{y} M$ to be the orthogonal projection of $\dot{\gamma}_{z}(1)$ onto $\left(\operatorname{span}\left\{w, w^{\prime}\right\}\right)^{\perp}$. Now define $h_{1}(z)=\left\|\pi\left(\dot{\gamma}_{z}(1)\right)\right\|_{g_{y}}$. For $z \in \partial M$, we have $h_{1}(z)=0$ if and only if $z \in Z$. Now let $\psi^{1} \in C_{0}^{\infty}(\mathbb{R})$ satisfy $0 \leq \psi^{1} \leq 1$, $\psi^{1}(0)=1, \int_{\mathbb{R}} \psi^{1}(x) d x=1$ and define $\psi_{\rho}^{1}: \partial M \rightarrow \mathbb{R}$ by

$$
\psi_{\rho}^{1}(z)=\psi^{1}\left(\frac{h_{1}(z)}{\rho}\right) ;
$$


$\psi_{\rho}^{1}$ concentrates at $Z$ as $\rho$ approaches 0 .

For $0 \leq s \leq \tau(y, w)$ let $\hat{y}(s)=\gamma_{(y, w)}\left(s-\tau_{-}(y, w)\right), \hat{b}(s)=\mathcal{P}\left(w^{\prime} ; y, \hat{y}(s)\right) \in$ $T_{\hat{y}(s)} M$, and denote $x^{*}=\gamma_{\left(y, w^{\prime}\right)}\left(-\tau_{-}\left(y, w^{\prime}\right)\right)$. See Figure 2 .

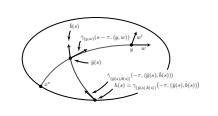

\section{Figure 2.}

Define $h(s)=\gamma_{(\hat{y}(s), \hat{b}(s))}\left(-\tau_{-}(\hat{y}(s), \hat{b}(s))\right) \in \partial M$. Note that $h\left(\tau_{-}(y, w)\right)=$ $x^{*}$. It is easily seen that $h(s) \in Z$ for each $s$, so $h_{1}(h(s))=0$. Denote by $w^{*} \in T_{x^{*}} \partial M$ the tangent vector

$$
\left.\frac{d}{d s}\right|_{s=\tau_{-}(y, w)} h(s)
$$

since $w$ and $w^{\prime}$ are linearly independent, it is readily checked that $w^{*} \neq 0$. Define $h_{2}: \partial M \rightarrow \mathbb{R}$ by

$$
h_{2}\left(x^{\prime}\right)=\left\langle\operatorname{Exp}_{x^{*}}^{-1}\left(x^{\prime}\right), w^{*}\right\rangle_{g_{x^{*}}}+\operatorname{sgn}\left(\left\langle\operatorname{Exp}_{x^{*}}^{-1}\left(x^{\prime}\right), w^{*}\right\rangle_{g_{x^{*}}}\right)\left\|\phi_{1}\left(x^{\prime}\right)-\phi_{1}\left(x^{*}\right)\right\|_{\mathbb{R}^{n}} \text {. }
$$

The function $h_{2}$ has the property that $h_{2}=0$ only at $x^{\prime}=x^{*}$. Furthermore, if $s_{0}=\tau_{-}(y, w)$ then

$$
\left.\frac{d}{d s}\right|_{s=s_{0}} h_{2}(h(s))=\left\|w^{*}\right\|_{g_{x^{*}}} \neq 0 .
$$

Now let $\psi^{2} \in C_{0}^{\infty}(\mathbb{R})$ satisfy $0 \leq \psi^{2} \leq 1, \psi^{2}(0)=1, \int_{\mathbb{R}} \psi^{2}(x) d x=1 /\left\|w^{*}\right\|$, and define $\psi_{\varepsilon}^{2}$ by

$$
\psi_{\varepsilon}^{2}(x)=\frac{1}{\varepsilon} \psi^{2}\left(\frac{x}{\varepsilon}\right) .
$$

We shall need one more approximate identity function. Let $\psi^{3} \in C_{0}^{\infty}\left(\mathbb{R}^{n}\right)$ satisfy $0 \leq \psi^{3} \leq 0, \psi^{3}(0)=1, \int_{\mathbb{R}^{n}} \psi^{3}(x) d x=1$, and define

$$
\psi_{\epsilon}^{3}(x)=\frac{1}{\epsilon^{n}} \psi^{3}\left(\frac{x}{\epsilon}\right) \text {. }
$$

Let

$W=\left\{\left(y, w, w^{\prime}\right) \in M \times T_{y} M \times T_{y} M \mid w\right.$ and $w^{\prime}$ are linearly independent $\}$. 
Proposition 4.3. If $n \geq 3$ and $\left(y, w, w^{\prime}\right) \in M \times T_{y} M \times T_{y} M$ with $w$ and $w^{\prime}$ linearly independent we have

$$
\begin{array}{r}
\lim _{\varepsilon \rightarrow 0} \lim _{\rho \rightarrow 0} \lim _{\epsilon \rightarrow 0} \int_{\Gamma_{-}} \psi_{\rho}^{1}\left(x^{\prime}\right) \psi_{\varepsilon}^{2}\left(f\left(x^{\prime}\right)\right) \psi_{\epsilon}^{3}\left(\phi_{2}\left(v^{\prime}\right)-\phi_{2}\left(\mathcal{P}\left(w^{\prime} ; y, x^{\prime}\right)\right)\right) \\
\cdot \alpha\left(\vec{\gamma}_{(y, w)}\left(\tau_{+}(y, w)\right), x^{\prime}, v^{\prime}\right) d \mu\left(x^{\prime}, v^{\prime}\right) \\
=E\left(\vec{\gamma}_{(y, w)}\left(\tau_{+}(y, w)\right), 0,-\tau_{+}(y, w)\right) E\left(\vec{\gamma}_{\left(y, w^{\prime}\right)}\left(\tau_{-}\left(y, w^{\prime}\right)\right), 0, \tau_{-}\left(y, w^{\prime}\right)\right) \\
\cdot k\left(y, w, w^{\prime}\right),
\end{array}
$$

where the limit holds in $L_{\mathrm{loc}}^{1}(W)$.

Proof. Replacing $\alpha$ by $\alpha_{0}$ and integrating with respect to $d \mu\left(x^{\prime}, v^{\prime}\right)$ we obtain a multiple of $\psi_{\varepsilon}^{2}\left(h_{2}\left(\gamma_{(y, w)}\left(-\tau_{-}(y, w)\right)\right)\right)$ that (for all sufficiently small $\varepsilon$ ) is zero unless $w$ and $w^{\prime}$ are linearly dependent.

Substituting $\alpha_{1}$ for $\alpha$, changing variables $(a, b)=\vec{\gamma}_{\left(x^{\prime}, v^{\prime}\right)}(r)$, and interchanging the order of integration from $d s d b_{a} d \omega(a)$ to $d b_{a} d \omega(a) d s$ we obtain

$$
\begin{gathered}
\int_{0}^{\tau(y, w)} \int_{M} \int_{T_{a} M} \psi_{\rho}^{1}\left(\gamma_{(a, b)}\left(-\tau_{-}(a, b)\right)\right) \psi_{\varepsilon}^{2}\left(h_{2}\left(\gamma_{(a, b)}\left(-\tau_{-}(a, b)\right)\right)\right) \\
\cdot \psi_{\epsilon}^{3}\left(\phi_{2}\left(\dot{\gamma}_{(a, b)}\left(-\tau_{-}(a, b)\right)\right)-\phi_{2}\left(\mathcal{P}\left(w^{\prime} ; y, \gamma_{(a, b)}\left(-\tau_{-}(a, b)\right)\right)\right)\right) \\
\cdot E(\cdot) E(\cdot) k\left(a, b, \dot{\gamma}_{(y, w)}\left(s-\tau_{-}(y, w)\right)\right) \delta_{\hat{y}(s)}(a) d b_{a} d \omega(a) d s \\
=\int_{0}^{\tau(y, w)} \int_{T_{\hat{y}} M} \psi_{\rho}^{1}\left(\gamma_{(\hat{y}, b)}\left(-\tau_{-}(\hat{y}, b)\right)\right) \psi_{\varepsilon}^{2}\left(h_{2}\left(\gamma_{(\hat{y}, b)}\left(-\tau_{-}(\hat{y}, b)\right)\right)\right) \\
\cdot \psi_{\epsilon}^{3}\left(\phi_{2}\left(\dot{\gamma}_{(\hat{y}, b)}\left(-\tau_{-}(\hat{y}, b)\right)\right)-\phi_{2}\left(\mathcal{P}\left(w^{\prime} ; y, \gamma_{(\hat{y}, b)}\left(-\tau_{-}(\hat{y}, b)\right)\right)\right)\right) \\
\cdot E(\cdot) E(\cdot) k\left(\hat{y}, b, \dot{\gamma}_{(y, w)}\left(s-\tau_{-}(y, w)\right)\right) d b_{\hat{y}} d s .
\end{gathered}
$$

Here $\hat{y}=\hat{y}(s)$. Now $\phi_{2}\left(\dot{\gamma}_{(\hat{y}, b)}\left(-\tau_{-}(\hat{y}, b)\right)\right)-\phi_{2}\left(\mathcal{P}\left(w^{\prime} ; y, \gamma_{(\hat{y}, b)}\left(-\tau_{-}(\hat{y}, b)\right)\right)\right)$ equals $\phi_{2}(b)-\phi_{2}\left(\mathcal{P}\left(w^{\prime} ; y, \hat{y}(s)\right)\right)$ since our global coordinates $\phi_{2}$ are obtained by parallel translation. Set $\mathcal{P}\left(w^{\prime} ; y, \hat{y}(s)\right)=\hat{b}(s) \in T_{\hat{y}(s)} M$. The expression of $\psi_{\epsilon}^{2}$ becomes $\psi_{\epsilon}^{2}\left(\phi_{2}(b)-\phi_{2}(\hat{b}(s))\right)$ and taking the limit as $\epsilon \rightarrow 0$ (in $L^{1}$ ) we obtain

$$
\int_{0}^{\tau(y, w)} \psi_{\varepsilon}^{2}\left(h_{2}(h(s))\right) E(\cdot) E(\cdot) k\left(\hat{y}(s), \hat{b}(s), \dot{\gamma}_{(y, w)}\left(s-\tau_{-}(y, w)\right)\right) d s .
$$

Set $\widetilde{s}=h_{2}(h(s))$; then

$$
\left.\frac{d \widetilde{s}}{d s}\right|_{s=\tau_{-}(y, w)}=\left\|w^{*}\right\| \neq 0
$$

and so for sufficiently small $\varepsilon$, for all $s$ in the support of $\psi_{\varepsilon}^{2}\left(h_{2}(h(s))\right)$ we have $\frac{d \widetilde{s}}{d s} \neq 0$ and we may perform the change of variables from $s$ to $\widetilde{s}$ in the 
above integral. Since $f\left(h\left(\tau_{-}(y, w)\right)\right)=0$, setting $s=(f \circ h)^{-1}(\widetilde{s})$ we obtain (for sufficiently small $\delta$ )

$$
\begin{aligned}
& \int_{-\delta}^{\delta} \psi_{\varepsilon}^{1}(\widetilde{s}) E(\cdot) E(\cdot) k\left(\hat{y}\left(s, \hat{b}(s), \dot{\gamma}_{(y, w)}\left(s-\tau_{-}(y, w)\right)\right) \frac{d s}{d \widetilde{s}} d \widetilde{s}\right. \\
& \rightarrow E(\cdot) E(\cdot) k\left(\hat{y}(s(0)), b(s(0)), \dot{\gamma}_{(y, w)}\left(s(0)-\tau_{-}(y, w)\right)\right)
\end{aligned}
$$

as $\varepsilon \rightarrow 0$, which in turn equals

$E\left(\vec{\gamma}_{(y, w)}\left(\tau_{+}(y, w)\right), 0,-\tau_{+}(y, w)\right) E\left(\vec{\gamma}_{\left(y, w^{\prime}\right)}\left(\tau_{-}\left(y, w^{\prime}\right)\right), 0, \tau_{-}\left(y, w^{\prime}\right)\right) k\left(y, w, w^{\prime}\right)$.

Finally, let $\chi\left(y, w, w^{\prime}\right) \in C_{0}^{\infty}(W)$. Let

$$
\begin{aligned}
& G_{-}=\left\{\left(x^{\prime}, v^{\prime}\right) \in \Gamma_{-} \mid \phi_{1}\left(v^{\prime}\right)-\phi_{1}\left(\mathcal{P}\left(w^{\prime} ; y, x^{\prime}\right)\right) \in \operatorname{supp} \psi_{\epsilon}^{3}, w^{\prime} \in \operatorname{supp} \chi\right\}, \\
& G_{+}=\left\{(x, v)=\vec{\gamma}_{(y, w)}\left(\tau_{+}(y, w)\right) \in \Gamma_{+} \mid(y, w) \in \operatorname{supp} \chi\right\} .
\end{aligned}
$$

Then

$$
\begin{aligned}
& \int_{M} \int_{T_{y} M} \int_{T_{y} M} \mid \int_{\Gamma_{-}} \psi_{\rho}^{1}\left(x^{\prime}\right) \psi_{\varepsilon}^{2}\left(h_{2}\left(x^{\prime}\right)\right) \psi_{\epsilon}^{3}\left(\phi_{2}\left(v^{\prime}\right)-\phi_{2}\left(\mathcal{P}\left(w^{\prime} ; y, x^{\prime}\right)\right)\right) \\
& \cdot \chi\left(y, w, w^{\prime}\right) \alpha_{2}\left(\vec{\gamma}_{(y, w)}\left(\tau_{+}(y, w)\right), x^{\prime}, v^{\prime}\right) d \mu\left(x^{\prime}, v^{\prime}\right) \mid d w_{y}^{\prime} d w_{y} d \omega(y) \\
& \leq \frac{1}{\varepsilon \epsilon} \int_{G_{+}} \int_{0}^{\tau_{-}(x, v)} \int_{T_{\gamma_{(x, v)}(s)} M} \int_{G_{-}} \psi_{\rho}^{1}\left(x^{\prime}\right) \chi\left(\vec{\gamma}_{(x, v)}(s), w^{\prime}\right) \alpha_{2}\left(x, v, x^{\prime}, v^{\prime}\right) \\
& \leq \frac{1}{\varepsilon \epsilon} \int_{G_{-}} \int_{G_{+}} \psi_{\rho}^{1}\left(x^{\prime}\right) C_{\chi}(x, v) \tau(x, v) \alpha_{2}\left(x, v, x^{\prime}, v^{\prime}\right) d \mu(x, v) d \mu\left(x^{\prime}, v^{\prime}\right)
\end{aligned}
$$

where

$$
C_{\chi}(x, v)=\sup _{s \in[0, \tau(x, v)]} \int_{T_{\gamma_{(x, v)}(s)} M} \chi\left(\vec{\gamma}_{(x, v)}(s), w^{\prime}\right) d w_{\gamma_{(x, v)}(s)}^{\prime} .
$$

The integrand on the last line of $(25)$ is an $L^{1}$ function since $\tau(x, v) C_{\chi}(x, v)$ is bounded for $(x, v) \in \operatorname{supp} C_{\chi}$, and $\alpha_{2} \in L^{\infty}\left(\Gamma_{-} ; L^{1}\left(\Gamma_{+}, d \mu\right)\right)$. Since the support of $\psi_{\rho}^{1}$ is a $\rho$-small neighborhood of a $3 n$-dimensional variety in the $4 n-2$-dimensional $\Gamma_{+} \times \Gamma_{-}$, the integral (25) tends to zero as $\rho \rightarrow 0$. This completes the proof of Proposition 4.3.

Theorem 4.4. If $M \subset \mathbb{R}^{n}, n \geq 3$, is a bounded domain with $C^{\infty}$ boundary, $g$ is a simple Riemannian metric on $M$, and assumptions (3) and (4) hold then from the kernel of $\mathcal{A}$ we may determine the collision kernel $k\left(x, v^{\prime}, v\right)$.

Proof. From Theorem 4.2 we can determine $\sigma_{a}$ from $\mathcal{A}$ and so determine $E(x, v, s, t)$; taking the limit as in Proposition 4.3, we can thus determine $k\left(x, v^{\prime}, v\right)$ for $v^{\prime}, v \in T_{x} M$ linearly independent. Of course $k$ is an $L^{1}$ function and so knowing $k$ on such a set of $v^{\prime}, v$ is equivalent to knowing $k$. 
Acknowledgements. The author would like to thank Gunther Uhlmann for suggesting this problem and for several helpful discussions. We also thank Simon Arridge for the reference $[\mathbf{F}]$.

\section{References}

[A] S. Arridge, Optical tomography in medical imaging, Inverse Problems, 15 (1999), R41-R93, MR 1684463 (2000b:78023), Zbl 0926.35155.

[BG] I.N. Bernstein and M.L. Gerver, Conditions of distinguishability of metrics by hodographs, Methods and Algorithms of Interpretation of Seismological Information, Computerized Seismology, 13 (1980), Nauka, Moscow, 50-73 (in Russian).

[CS1] M. Choulli and P. Stefanov, Inverse scattering and inverse boundary value problems for the linear Boltzmann equation, Comm. Partial Differential Equations, 21(5-6) (1996), 763-785, MR 1391523 (97f:35230), Zbl 0857.35131.

[CS2] M. Choulli and P. Stefanov, An inverse boundary value problem for the stationary transport equation, Osaka J. Math. 36(1) (1999), 87-104, MR 1670750 (2000g:35228), Zbl 0998.35064.

[DU] J. Diestel and J.J. Uhl, Jr., Vector Measures, Mathematical Surveys and Monographs, 15, American Mathematical Society, Providence, R.I., 1977, MR 0453964 (56 \#12216), Zbl 0369.46039.

[F] H.A. Ferwerda, The radiative transfer equation for scattering media with a spatially varying refractive index, J. Opt. A: Pure Appl. Opt., 1 (1999), L1-L2.

$[\mathrm{H}] \quad$ S. Helgason, Differential Geometry, Lie Groups and Symmetric Spaces, Pure and Applied Mathematics, 80, Academic Press, New York, 1978, MR 0514561 (80k:53081), Zbl 0451.53038.

$[\mathrm{KH}]$ A. Katok and B. Hasselblatt, Introduction to the Modern Theory of Dynamical Systems, Encyclopedia of Mathematics and its Applications, 54, Cambridge University Press, Cambridge, 1995, MR 1326374 (96c:58055), Zbl 0878.58020.

[M] R.G. Mukhometov, On a problem of reconstructing Riemannian metrics, Siberian Math. J., 22(3) (1982), 420-433, MR 0621466 (82m:53071), Zbl 0478.53048.

[RS] M. Reed and B. Simon, Methods of Modern Mathematical Physics, III: Scattering Theory, Academic Press, New York, 1979, MR 0529429 (80m:81085), Zbl 0405.47007.

[R] V.G. Romanov, Stability estimates in problems of recovering the attenuation coefficient and the scattering indicatrix for the transport equation, J. Inverse Ill-Posed Probl. 4(4) (1996), 297-305, MR 1403885 (97f:35235), Zbl 0860.35146.

[Sh1] V.A. Sharafutdinov, Integral Geometry of Tensor Fields, Inverse and Ill-Posed Problems Series, VSP, The Netherlands, 1994, MR 1374572 (97h:53077), Zbl 0883.53004.

[Sh2] V.A. Sharafutdinov, The inverse problem of determining the source in the stationary transport equation on a Riemannian manifold, J. Math. Sci. (New York), 96(4) (1999), 3430-3433, MR 1700653 (2000g:58056), Zbl 0935.58013.

[SU] P. Stefanov and G. Uhlmann, Optical tomography in two dimensions, Methods Appl. Anal., 10(1) (2003), 1-9, MR 2014159 (2004h:35224).

[T1] A. Tamasan, Optical tomography in weakly anisotropic scattering media, in Inverse problems: theory and applications (Cortona/Pisa, 2002), Contemp. Math. 333, Amer. Math. Soc., Providence, RI, 2003, 199-207, MR 2032017. 
[T2] A. Tamasan, An inverse boundary value problem in two-dimensional transport, Inverse Problems, 18(1) (2002), 209-219, MR 1893591 (2003h:35283), Zbl 0995.65146.

[W] J.-N. Wang, Stability estimates of an inverse problem for the stationary transport equation, Ann. Inst. H. Poincaré Phys. Théor., 70(5) (1999), 473-495, MR 1697917 (2000e:82042), Zbl 0963.35204.

Received July 30, 2003 and revised April 2, 2004. This project was partially funded by NSF Grants DMS-9970503, DMS-0070488.

Department of Mathematics

WESTERN WASHINGTON UNIVERSITY

Bellingham WA 98225-9063

E-mail address: stephen.mcdowall@wwu.edu 